\title{
Battage de pieux métalliques dans la roche ${ }^{\text {tr }}$
}

\author{
Alain Holeyman ${ }^{\star}$ \\ Université catholique de Louvain, place du Levant, 1 à 1348 Louvain-la-Neuve, Belgique
}

\begin{abstract}
Résumé - Le problème du battage d'un pieu tubulaire en acier dans un massif rocheux est abordé sous deux volets: numérique et expérimental. L'approche numérique par éléments finis couplant une modélisation lagrangienne du pieu en mouvement à une modélisation eulérienne du massif en place (approche CEL) permet de suivre l'émergence de la plastification dans le tube en acier en cours de pénétration, naissant en pointe pour se propager vers la tête du pieu. Un cas d'étude en conditions axisymétriques est présenté pour une roche dont la résistance à la compression simple $\sigma_{c}$ vaut $28 \mathrm{MPa}$. L'approche expérimentale poursuivie en laboratoire a mis en jeu trois matériaux synthétisés sous la forme de monolithes dans lesquels le battage d'un tube en acier inoxydable a été entrepris. Les résultats de ces essais largement instrumentés indiquent que le battage est aisé dans un mortier cellulaire dont la résistance à la compression simple $\sigma_{c}$ n'excède pas $6 \mathrm{MPa}$ mais pratiquement impossible dans un mortier dont la résistance à la compression simple s'approche de $28 \mathrm{MPa}$. Le battage dans un mortier dont la résistance à la compression simple vaut $11 \mathrm{MPa}$ s'est révélée encore faisable sous une hauteur de chute raisonnable. Les résultats obtenus avec les 3 matériaux mis en œuvre à ce jour indiquent que la résistance unitaire croît depuis environ $2 \sigma_{c}$ en surface pour atteindre 6 à $8 \sigma_{c}$ à une pénétration équivalente à 15-20 fois l'épaisseur du tube.
\end{abstract}

Mots clés : pieu / roche / tube / Hoek-Brown / Drucker-Prager étendu / couplage eulérien-lagrangien (CEL) / modèle de laboratoire / endommagement

\begin{abstract}
Steel pile driving into rock. The problem of driving a steel pipe pile in rock is addressed in two ways: numerical and experimental. The numerical approach by finite elements coupling Lagrangian elements modeling the moving pile and Eulerian elements modeling the rock in place (CEL approach) allows one to follow the emergence of the steel tube plastification in the course of penetration, starting from the pile toe and propagating upwards. A case study in axisymmetric conditions is presented for a rock with an unconfined compressive strength $\sigma_{c}$ worth $28 \mathrm{MPa}$. The experimental approach pursued in the laboratory features three materials synthesized in the form of monoliths in which the driving of a stainless steel tube has been undertaken. The results of these heavily instrumented driving tests indicate that pipe pile driving is easy in a cellular mortar with an unconfined compression resistance $\sigma_{c}$ not exceeding $6 \mathrm{MPa}$ but virtually impossible in a mortar with an unconfined compression resistance $\sigma_{c}$ approaching $28 \mathrm{MPa}$. Driving into a mortar with an unconfined compression resistance $\sigma_{c}$ of $11 \mathrm{MPa}$ proved to be still feasible under a reasonable drop height. The results obtained with the 3 materials implemented to date indicate that the unit toe resistance increases from approximately $2 \sigma_{C}$ at the rock surface to reach 6 to $8 \sigma_{C}$ at a penetration equivalent to 15-20 times the thickness of the tube.
\end{abstract}

Keywords: pile / rock / tube / Hoek-Brown / extended Drucker-Prager / coupled Eulerian-Lagrangian (CEL) / laboratory model / damage

\section{Introduction}

Le marché éolien est en pleine expansion mondiale et son secteur offshore offre des perspectives très intéressantes,

\footnotetext{
Article introduit à la Revue française de Géotechnique en support à la Conférence Coulomb 2017 intitulée «Comportement axial des pieux sous sollicitations dynamiques extrêmes »

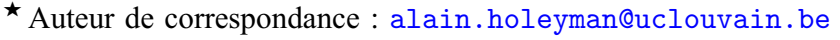

surtout lorsque les nuisances de l'éolien on-shore sont prises en compte. À titre d'exemple, même pour un pays comme la Belgique qui ne possède que peu d'eaux territoriales, les Autorités estiment qu'en 2020 plus de $60 \%$ de la production électrique éolienne belge proviendra de l'offshore. Certains développements technologiques récents permettent de penser que la barre des $10 \mathrm{MW}$ sera prochainement atteinte par les éoliennes. On comprendra que les exigences vis-à-vis des fondations s'accroissent en termes de capacité portante et de profondeur au fur et à mesure que la puissance des éoliennes croît. 

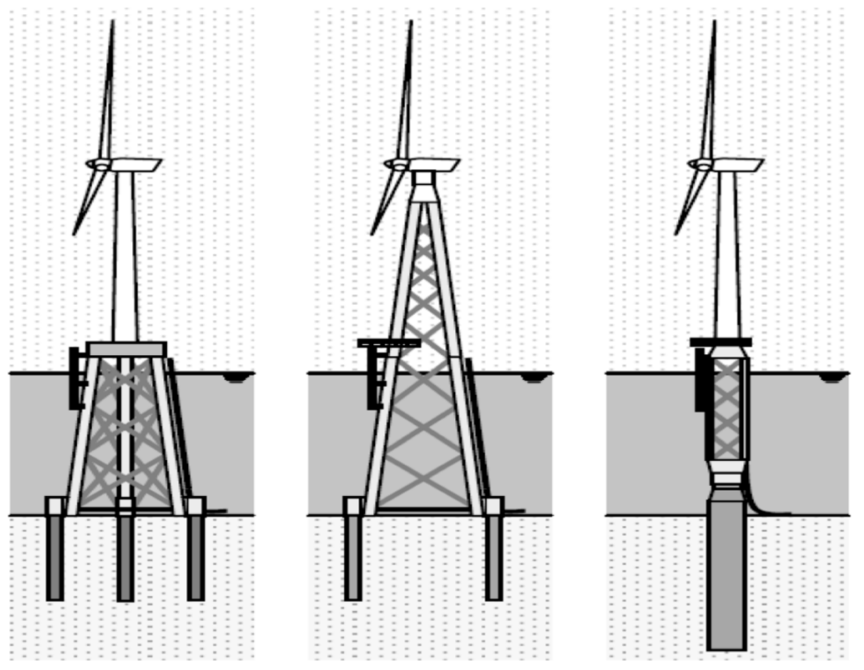

Fig. 1. Utilisations typiques de pieux pour supporter des éoliennes off-shore: Tripode, Jacket et Monopieu (Upwind, 2010).

Fig. 1. Typical use of piles to support wind turbines: Tripod, Jacket, and Monopile (Upwind, 2010).

L'un des principaux inconvénients des parcs éoliens offshore est lié aux impératifs techniques complexes de leur mise en œuvre, ce qui entraîne un coût de mise en service beaucoup plus important. On estime que les frais d'installation s'élèvent à $30 \%$ du prix global de l'éolienne alors qu'une installation offshore est de 2 à 4 fois plus chère qu'une éolienne on-shore. L'offshore permet toutefois d'installer des unités plus grandes et dont le facteur de charge est environ $50 \%$ plus élevé. En outre, les techniques actuellement appliquées sont généralement complexes et nécessitent des adaptations parfois difficiles aux impératifs de terrain. Il en résulte un risque important qui se répercute, soit en termes de frilosité vis-à-vis de certains sites, soit en surcoût à charge de la communauté.

Les coûts d'installation des éoliennes offshore devraient être non seulement diminués, mais surtout maîtrisés. La pression que la société s'impose actuellement pour développer des énergies nouvelles pose donc de nouveaux défis à l'ingénierie des fondations profondes. La question du battage des pieux tubulaires en acier dans des formations géologiques indurées est à résoudre maintenant si l'on veut installer des éoliennes de grand calibre au large.

Les pieux sont largement utilisés dans des ouvrages de génie civil lorsque d'importantes charges se retrouvent concentrées en quelques points. C'est exactement le cas des éoliennes supportées en altitude par un mât soumis à des actions latérales importantes principalement dues aux vents et aux vagues. Des cas typiques de fondations d'éoliennes faisant intervenir des pieux sont illustrés sur la figure 1. Leur fonction est de transmettre les efforts statiques et variables, voire cycliques, provenant du mât de support vers les couches résistantes en profondeur.

On peut anticiper que les cas de charge sous conditions extrêmes de tempête appliqués à des éoliennes de plus en plus grandes exigent des fondations de plus en plus profondes dans des terrains meubles. Dans le cas où les conditions géologiques offrent des bancs rocheux à faible profondeur, une fonction d'ancrage à profondeur modérée est souhaitable pour assurer la stabilité de l'installation vis-à-vis du renversement.
Comme les formations indurées ont été traditionnellement considérées comme inabordables par battage, il est nécessaire d'approcher ce nouveau problème au moyen de différentes approches. L'objet de cet article en support de la Conférence Coulomb 2017 est de présenter quelques développements numériques et expérimentaux récemment menés au sein de l'équipe de recherche géotechnique de l'Université catholique de Louvain en Belgique visant à résoudre ce défi.

La Conférence Coulomb 2017 suivra un cheminement qui se veut didactique en commençant par les éléments théoriques permettant de maîtriser les éléments essentiels d'un calcul de battage de pieu, en inspectant les révélations apportées par une modélisation numérique, et en finissant par les questions complémentaires révélées par une expérimentation sur modèle réduit, afin de se recentrer sur l'enjeu in situ.

\section{Le défi du battage}

\subsection{Enjeux}

Le battage d'un pieu dans le sol consiste à lui asséner des coups au moyen d'une masse frappante afin qu'il pénètre sans endommagement dans le sol. L'art de cette technologie au principe élémentaire, pour ne pas dire barbare, réside dans la modulation de l'impact, tant en amplitude qu'en durée, afin de vaincre la résistance du sol mais sans toutefois dépasser la résistance structurale du pieu à installer. $\mathrm{La}$ méthode alternative du vibro-fonçage décrite par Holeyman et Whenham $(2015,2017)$ ne fait pas l'objet de cet article.

La figure 2 présente les principaux acteurs intervenant lors du battage: le pieu, le géomatériau, le mouton ainsi que les questions que leurs interactions suscitent. Si les dimensions des pieux concernés par les projets offshore sont imposantes (depuis typiquement $1,8 \mathrm{~m}$ de diamètre pour un pieu de fondation d'une structure de type jacket jusqu'actuellement $9 \mathrm{~m}$ de diamètre pour un mono-pieu), la durée de l'impact reste réduite, typiquement de l'ordre d'un centième de seconde. L'enfoncement du pieu qui résulte de cette action transitoire passe par un maximum pour revenir, après rebond, à l'enfoncement permanent, résultat attendu de chaque coup en termes de pénétration du pieu.

Le dimensionnement géotechnique du pieu (diamètre et profondeur) est régi par des considérations principalement statiques afin d'assurer la stabilité de l'ouvrage. Le concepteur recherche en premier lieu la sécurité des fondations compte tenu des actions auxquelles est soumis l'ouvrage. Nombre de prescriptions codées ou normalisées (API, 2011 ; Eurocode 7, NF, 2005, 2006, 2012) détaillent les différentes vérifications permettant de garantir cette stabilité, sans que ces documents fournissent pour autant de l'inspiration au concepteur.

Pour des réalisations sur la terre ferme, le problème de l'exécution et en particulier de l'installation de pieux, demeure la principale préoccupation de l'entreprise. En revanche, le concepteur de pieux offshore doit anticiper l'éventuelle difficulté que l'entreprise pourrait rencontrer à installer les pieux jusqu'à la profondeur dimensionnée. La raison de cette différence réside dans la conséquence d'être confronté à une infaisabilité : elle se chiffre rapidement en millions d'euros. Les Maîtres d'ouvrage de tels projets exigent que ce risque soit maîtrisé. 


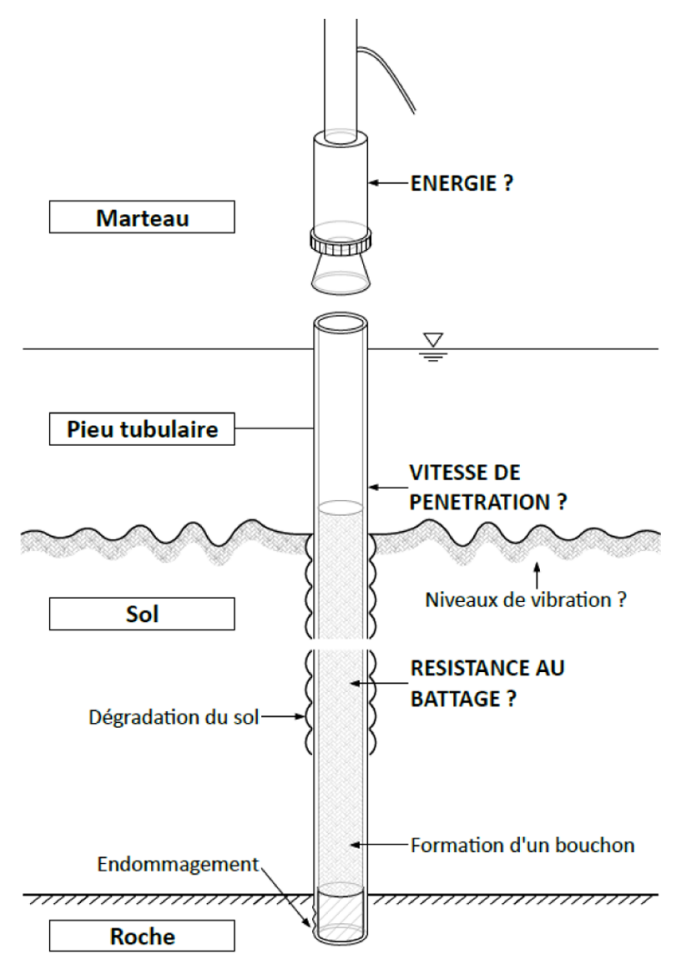

Fig. 2. Battage : acteurs et enjeux.

Fig. 2. Impact Driving: Players and Issues.

À la recherche de sites éoliens productifs au large, le concepteur est maintenant obligé de trouver des solutions économiques de fondations capitalisant sur la bonne résistance de couches indurées. Il est clair que l'économie d'échelle favorise par ailleurs des ouvrages éoliens d'envergure de plus en plus grande, requérant des fondations de plus en plus profondes, qui, même si elles ne peuvent tirer parti des couches indurées trop minces, doivent néanmoins les traverser pour atteindre la capacité portante désirée.

Il est maintenant devenu clair que la question du battage des pieux dans la roche ne peut plus être éludée.

Le principal enjeu soulevé par cette installation brutale est la survie structurale du pieu. Si dans le passé cette dernière pouvait être mise en danger en tête de pieu par un impact excessif, les progrès engrangés au cours des dernières décennies dans la maîtrise des calculs d'impact tendent à actuellement éviter cet écueil. L'impasse rémanente se présente dans une situation dite de «refus», à savoir que la résistance du sol lors du battage ne permet plus au pieu de pénétrer.

L'endommagement structural du pieu peut être évité grâce, non seulement aux progrès atteints dans la maîtrise des calculs de battage, mais aussi au fait que la résistance unitaire à la pénétration en pointe du pieu reste sensiblement inférieure à la limite élastique de l'acier constituant le pieu. À titre d'exemple, il est rare de rencontrer une résistance unitaire au cône de pénétration supérieure à $100 \mathrm{MPa}$ dans les sables les plus compacts alors que les aciers à haute performance utilisés en fondation offshore sont caractérisés par une limite élastique de 355 à $460 \mathrm{MPa}$.

Il n'en va plus de même pour des formations indurées (calcarénite...) rencontrées au large de certaines zones côtières européennes pour lesquelles la résistance théorique à la pénétration pourrait facilement dépasser $200 \mathrm{MPa}$ si elle pouvait être mesurée en pratique. Cette situation se rapproche du domaine où le burinage prévaut, caractérisé par une très faible pénétration par coup et donc le nombre de coups élevé. Des considérations de fatigue devront alors forcément entrer en jeu.

Le pieu pourra conserver son intégrité si aucun de ses modes de ruine structurale n'est atteint au cours du battage jusqu'à la profondeur de conception. Alors qu'il sera nécessaire d'inclure des phénomènes d'ovalisation, voire d'instabilité élastique par voilement de pieux tubulaires dans une analyse complète, l'objet de la présente communication se focalise sur les contraintes axiales, qu'elles soient moyennes sur la section du pieu ou localisées.

\section{2 Équation d'onde et éléments finis}

S'il a fallu attendre le milieu du $\mathrm{XX}^{\mathrm{e}}$ siècle pour comprendre que le battage d'un pieu pouvait s'analyser au départ des ondes qui le parcouraient (Isaacs, 1931), l'équation fondamentale à utiliser datait de deux siècles auparavant (D'alembert, 1747; De Saint Venant, 1867). Cette équation différentielle aux dérivées partielles (EDP) dénommée «équation d'onde» a fait l'objet de nombreuses publications, et même d'une série de conférences internationales ( Stress Wave Conference», allant de 1980 à nos jours).

On supposera que le lecteur est familier avec cette équation et ses applications et qu'il maîtrise entre autres les notions d'impédance du pieu, de réflexion aux conditions limites, ainsi que les effets de l'interaction de l'onde avec la réaction mobilisée le long du fût du pieu (Holeyman et al., 2013) ainsi qu'à sa pointe (Holeyman, 1988). Bien qu'il existe des solutions analytiques très utiles à cette équation (Holeyman, 1992), la pratique actuelle consacre le recours aux résolutions numériques (Smith, 1960), facilitées par la commercialisation de logiciels. Un des résultats le plus pertinent obtenu par l'équation d'onde est le doublement de la contrainte en pointe d'un pieu dont le mouvement est parfaitement empêché.

Si la modélisation numérique par éléments finis permet de simuler des applications géotechniques classiques, elle s'est avérée ardue pour simuler l'installation de pieux. Plusieurs chercheurs ont dû l'agrémenter de procédures très particulières afin d'éviter la distorsion excessive du maillage lagrangien. À titre d'exemple, Henke et Grabe (2008) ont élaboré un modèle axisymétrique fondé sur une technique dénommée « fermeture éclair» qui permet de modéliser le refoulement du sol par le pieu.

L'approche Couplée Eulérienne-Lagrangienne (CEL) disponible dans le logiciel commercial ABAQUS est une méthode alternative basée sur la combinaison d'éléments finis alliant les avantages des formulations lagrangienne et eulérienne pour définir la cinématique de corps déformables. Cette approche permet à des objets déformables en mouvement d'interagir avec un milieu continu globalement stationnaire, ainsi que publié par Qiu et al. (2011) et Jablonski et al. (2012).

Dans ce qui suit, l'approche CEL est utilisée pour simuler la pénétration dynamique d'un pieu tubulaire dans un sable dense recouvrant un banc de roche. Le pieu doit être conçu en fonction de l'évaluation fiable des contraintes au sein de 
l'acier générées au cours du battage. Quelques résultats sont présentés pour illustrer l'influence de la résistance de la couche de roche rencontrée et de la qualité de l'acier sur le maintien de l'intégrité du pieu tubulaire lors de son battage.

\section{Approche numérique}

\subsection{Critères de rupture de la roche}

Le critère de rupture de Hoek-Brown $(\mathrm{H}-\mathrm{B})$ est largement accepté pour caractériser le comportement de la roche dans sa masse et a été appliqué dans un grand nombre de projets à travers le monde. Hoek et Brown $(1980,1988)$ et Hoek et al. (2002) ont introduit leur critère de rupture en vue de faciliter la conception des excavations souterraines dans la roche compétente. Le critère provient d'une combinaison de résultats expérimentaux de recherche sur la rupture fragile de la roche intacte menée par Hoek et de résultats de modélisations du comportement de la masse rocheuse entreprises par Brown. Le critère se base sur les propriétés de la roche intacte en y intégrant nombre de facteurs réducteurs reflétant les faiblesses structurelles (joints, fissures...) affectant la masse de roche. Le critère H-B peut être exprimé comme suit :

$$
\sigma_{1}=\sigma_{3}+\sigma_{c i}\left(m_{b} \frac{\sigma_{3}}{\sigma_{c i}}+s\right)^{\alpha},
$$

où $\sigma_{1}$ et $\sigma_{3}$ sont les contraintes effectives principales de compression, respectivement majeure et mineure, $\sigma_{c i}$ est la résistance à la compression simple de la roche intacte, $m_{i}$ est une constante du matériau intact, tandis que $m_{b}, \alpha$, et $s$ sont des constantes de matériaux ( $s=1$ pour le cas de la roche intacte) qui peuvent être exprimées en fonction de l'indice de résistance géotechnique (GSI, pour Geotechnical Strength Index, Marinos et al., 2005) et le facteur de perturbation (D) comme suit :

$$
\begin{gathered}
m_{b}=m_{i} e^{\left(\frac{G S I-100}{28-14 D}\right)}, \\
s=e^{\left(\frac{G S I-100}{9-3 D}\right)}, \\
\alpha=\frac{1}{2}+\frac{1}{6}\left(e^{\frac{-G S I}{15}}-e^{\frac{-100}{15}}\right) .
\end{gathered}
$$

On notera que la valeur de $\alpha$ est fort proche de 0,5 et que l'équation (1) se rapproche d'une forme parabolique. Le critère $\mathrm{H}$-B exprimé à l'origine pour des conditions triaxiales $\left(\sigma_{2}=\sigma_{3}\right)$ peut être généralisé moyennant l'utilisation d'invariants du tenseur des contraintes:

$$
\begin{gathered}
p=I_{1}=\frac{\sigma_{1}+\sigma_{2}+\sigma_{3}}{3} \\
\mathrm{q}=\sqrt{3 J_{2}}=\sqrt{\frac{\left(\sigma_{1}-\sigma_{2}\right)^{2}+\left(\sigma_{2}-\sigma_{3}\right)^{2}+\left(\sigma_{3}-\sigma_{1}\right)^{2}}{2}}
\end{gathered}
$$

où $\sigma_{2}$ est la contrainte principale intermédiaire. En adoptant les notations classiques $p$ et $q$ pour exprimer respectivement la contrainte moyenne et la contrainte de Von Mises, Priest (2005) a suggéré la version 3D suivante du critère :

$$
\frac{1}{\sigma_{c i}} q^{2}+\frac{1}{3} m_{b} q-m_{b} p-s \sigma_{c i}=0 .
$$

Le critère étendu de Drucker-Prager (EDP), disponible sous la forme d'un «matériau» dans la bibliothèque de matériau $\mathrm{ABAQUS}{ }^{\circledR}$, est propre à représenter un critère de type Hoek-Brown. Ce matériau peut être utilisé pour deux types d'analyse: explicite (ABAQUS/explicit) intégrant le temps pas à pas et implicite (ABAQUS/Standard) recherchant un équilibre statique. Il est souvent utilisé pour simuler autant les sols granulaires que les roches. En particulier, le critère de rupture EDP s'exprime sous une forme de fonction puissance comme suit (Abaqus, 2014):

$$
F(p, q)=a q^{b}-p-p_{t}
$$

Le critère de rupture sous forme puissance de 1'EDP est représenté dans le plan $p-q_{t}$ en figure 3 , identifiant $-p_{t}$ comme limite de traction isotrope du matériau. Les paramètres du critère EDP peuvent être choisis afin de caler l'équation (7) sur le critère $\mathrm{H}-\mathrm{B}$ (Eq. (1)). Une détermination explicite des paramètres $a$, $b$, et $p_{t}$ a même été développée par Hamdi et Holeyman (2015) en imposant que les deux critères coïncident en trois points particuliers, judicieusement sélectionnés sur base du rapport $\eta=\mathrm{q} / \mathrm{p}$. Grâce à la sélection de trois points $\left(p_{A}=-s . \sigma_{c i} / m_{b}\right.$ et $\left.q_{A}=0 ; q_{B}=h_{B} \cdot p_{B} ; q_{C}=h_{C} \cdot p_{C}\right)$, les paramètres $p_{t}, b$ et $a$ peuvent être explicités comme suit:

$$
\begin{aligned}
p_{t} & =\frac{s \sigma_{c i}}{m_{b}} \quad b=\ln \left(\frac{p_{\eta_{B}}+p_{t}}{p_{\eta_{C}}+p_{t}}\right) / \ln \left(\frac{q_{\eta_{B}}}{q_{\eta_{C}}}\right) a \\
& =\frac{p_{\eta_{B}}+p_{t}}{q_{\eta_{B}}^{b}} .
\end{aligned}
$$

À titre d'exemple, la figure 3 compare dans le plan $p$ - $q$ les critères de rupture $\mathrm{H}-\mathrm{B}$ et EDP pour une masse rocheuse de très bonne qualité dont les propriétés recommandées par Hoek et Brown (1997) y sont reprises en encart. L'écart entre les deux formulations $\mathrm{H}-\mathrm{B}$ et EDP est à peine décelable. Pour un comportement élasto-plastique, des valeurs du module de Young (E) et du coefficient de Poisson ( $v$ ) doivent être adoptées.

Une fois rompue, la roche de caractère fragile possède encore une résistance résiduelle qui peut se modéliser par un critère de rupture de type Mohr-Coulomb ou en faisant appel, ainsi que suggéré par Ribacchi (2000), à des paramètres H-B réduits, par exemple $s^{r}=0.04 s$ et $m_{b}^{r}=0.65 m_{b}$.

\subsection{Cas d'étude}

Un secteur de $90^{\circ}$ du problème axisymétrique a été modélisé par Hamdi (2016) comme montré sur la figure 4. À cette fin, des conditions aux limites générales axisymétriques en termes de déplacements et de rotation sont prescrites selon les plans $X=0$ et $Y=0$. Des éléments eulériens tridimensionnels à 8 nœuds (EC3D8R) sont utilisés pour le sol et la roche alors que des éléments lagrangiens de type continuum C3D8R 

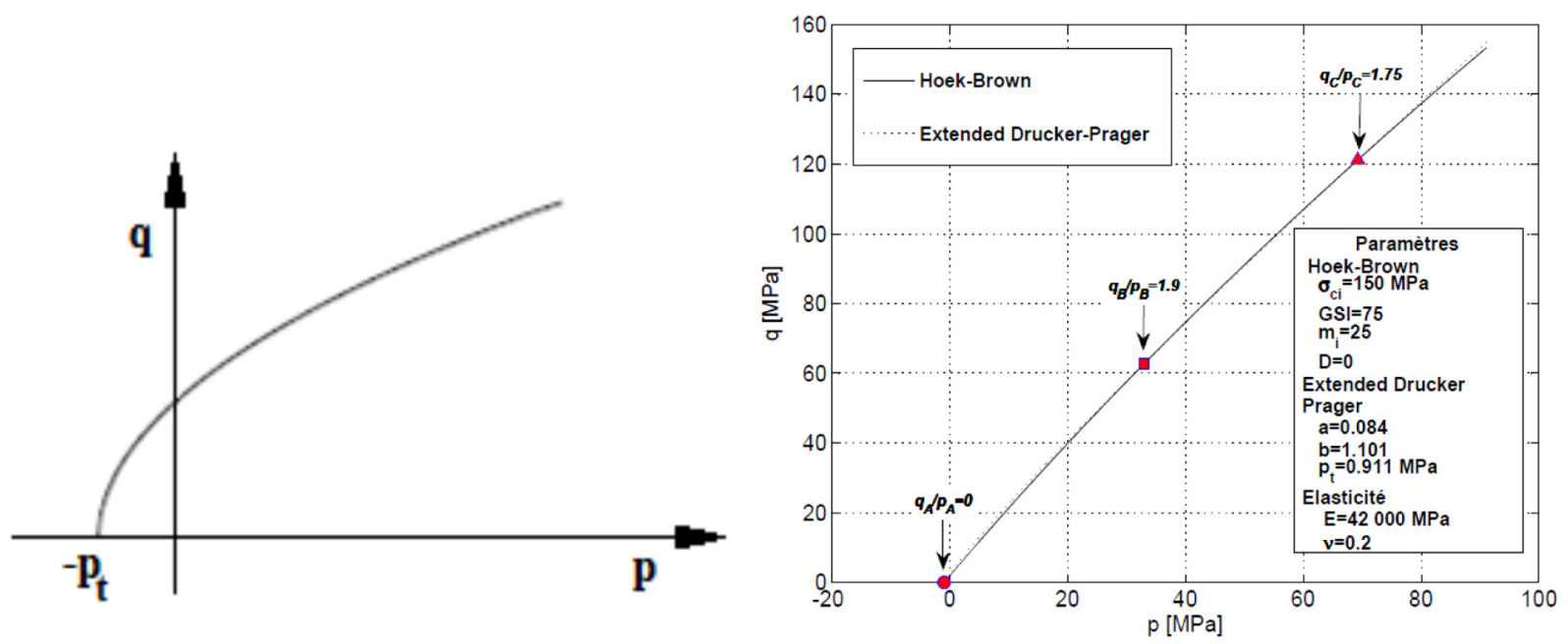

Fig. 3. Critères de rupture H-B et EDP dans le plan p-q : a) principe, b) cas d'une masse rocheuse de très bonne qualité (Hamdi et Holeyman, 2015). Fig. 3. Yield criterion in $p-q$ plane for both H-B and EDP: a) principle, b) case of a very good rock mass quality (Hamdi and Holeyman, 2015).

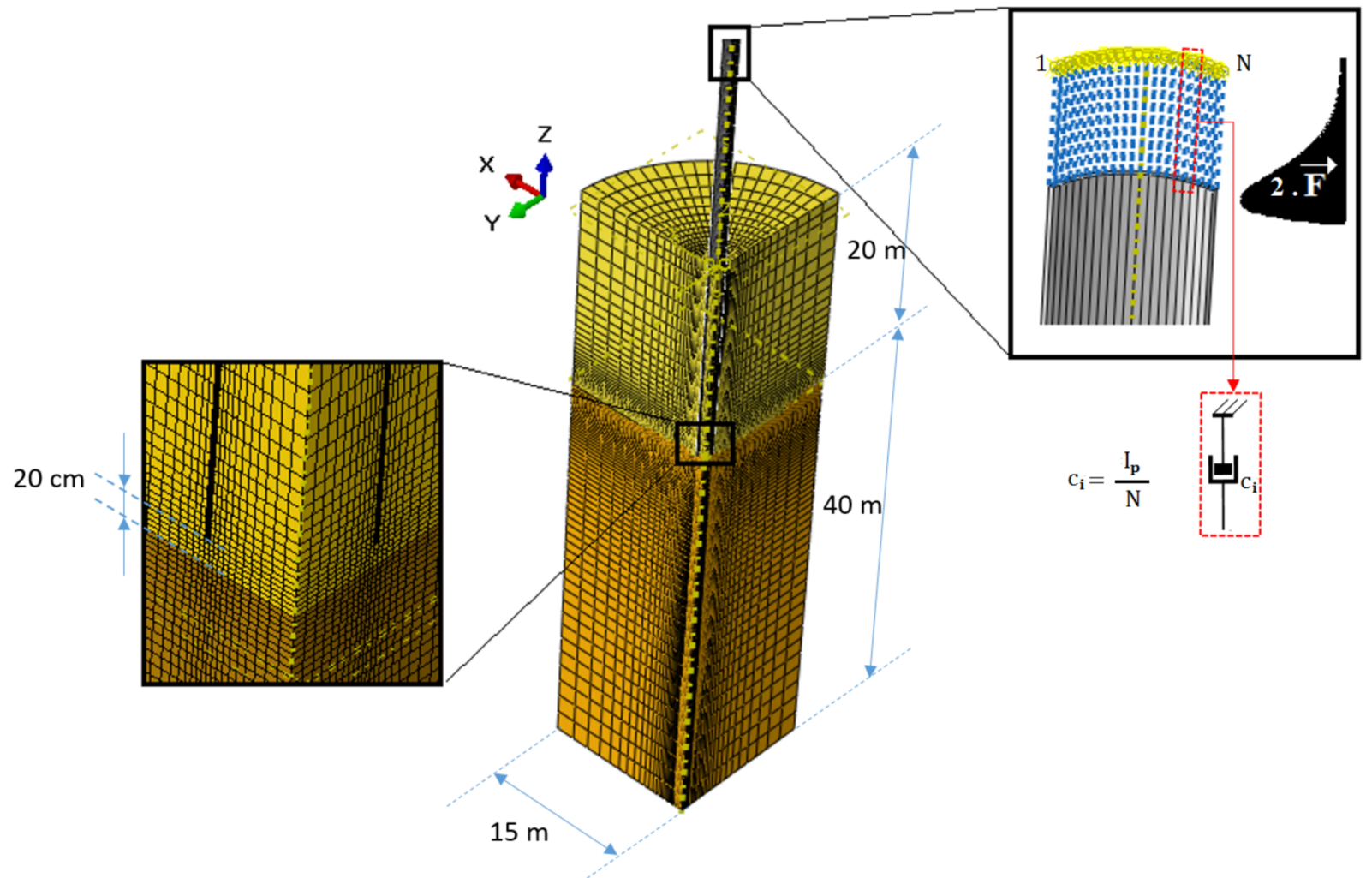

Fig. 4. Modélisation CEL et maillage : conditions aux limites (Hamdi, 2016).

Fig. 4. CEL modeling: meshing and boundary conditions (Hamdi, 2016).

(brique linéaire 8 nœuds à intégration réduite) modélisent le pieu tubulaire. Afin d'évaluer précisément les contraintes au sein du tube pendant le battage, son épaisseur est discrétisée en 8 éléments.

Le contact entre les sous-ensembles eulérien et lagrangien est géré à l'aide d'une formulation générale d'interpénétration pénalisante basée sur un concept de frontière «immergée».
Les éléments lagrangiens peuvent se déplacer au travers du maillage eulérien sans résistance jusqu'à ce qu'ils rencontrent un élément eulérien rempli de géomatériau.

Pour l'analyse de la propagation des ondes mécaniques au travers des sols et des roches en cours de battage, des conditions aux limites classiques (Dirichlet, Neumann) ne peuvent être utilisées aux frontières du domaine modélisé car 
Tableau 1. Paramètres H-B et EDP pour une masse rocheuse de très bonne qualité.

Table 1. H-B and EDP parameters for a very good rock quality mass.

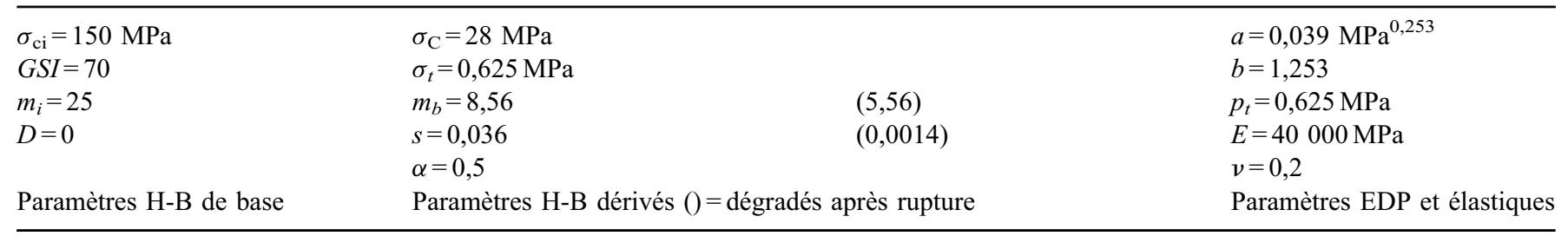

elles induisent la réflexion des ondes incidentes et rendent le calcul inexact. Afin de surmonter ce problème, des conditions aux limites dites «non-réfléchissantes » sont utilisées.

Les paramètres $\mathrm{H}-\mathrm{B}$ et EDP de la roche de très bonne qualité mise en jeu sont consignés dans le tableau 1. En ce qui concerne le sable dense surplombant la roche, les paramètres suivants d'un modèle élasto-plastique avec critère de rupture Mohr-Coulomb ont été adoptés : cohésion $c^{\prime}=0 \mathrm{kPa}$, angle de frottement interne $\varphi^{\prime}=40^{\circ}$, poids volumique effectif $\gamma^{\prime}=10,4 \mathrm{kN} / \mathrm{m}^{3}, \mathrm{E}=70 \mathrm{MPa}$ et $\nu=0,35$.

Pour le cas discuté ici, un marteau hydraulique de type IHC S-1200 a été utilisé pour simuler l'installation d'un pieu tubulaire de $1830 \mathrm{~mm}$ de diamètre $D$ et de $50,8 \mathrm{~mm}$ d'épaisseur $t(D / t=36)$ dans la roche. L'impact généré par ce marteau sur un pieu semi-infini suit la fonction mathématique type suivante (Holeyman, 1984) :

$$
F(t)=\text { C.t. } e^{-\alpha t}
$$

Afin d'éviter toute réflexion d'onde en tête de pieu où le signal doit être imposé, un ensemble d'amortisseurs sont connectés à la tête du pieu pour y simuler l'impédance de la partie supérieure du pieu. Comme la sollicitation est appliquée en une section courante d'un pieu virtuellement infini, une force d'amplitude double est prescrite (Fig. 4). Le signal de contrainte axiale descendante générée au niveau de la tête du pieu par un coup de marteau est représenté dans la figure 5. Il y correspond une énergie délivrée de $1200 \mathrm{~kJ}$.

Une série de coups de marteau a été appliquée au pieu tubulaire initialement fiché dans le sable à une profondeur de $20,0 \mathrm{~m}$. Avant d'atteindre la couche rocheuse située à une profondeur de 20,2 $\mathrm{m}$, le pieu pénètre d'abord à travers les $0,2 \mathrm{~m}$ restant de sable avant d'entrer en contact avec la masse rocheuse sous-jacente.

\subsection{Résultats numériques}

La figure 6 montre la pénétration du pieu sous une vingtaine de coups de marteau dans le cas d'une masse rocheuse de très bonne qualité (Tab. 1) située sous le sable moyennement compact. Le temps de repos entre les coups est numériquement raccourci afin de montrer les parties les plus intéressantes de la séquence des coups. L'enfoncement par coup est d'environ $4 \mathrm{~cm}$ dans le sable dense et d'environ $2 \mathrm{~cm}$ dans la couche de roche. Après chaque enfoncement maximum transitoire, un léger rebond du pieu est observé. En fait, la résistance au frottement latéral négativement mobilisé dans la couche de sable dense lors du rebond en limite l'amplitude.

L'estimation des contraintes au sein du tube en acier en cours de battage est essentielle pour en évaluer l'intégrité

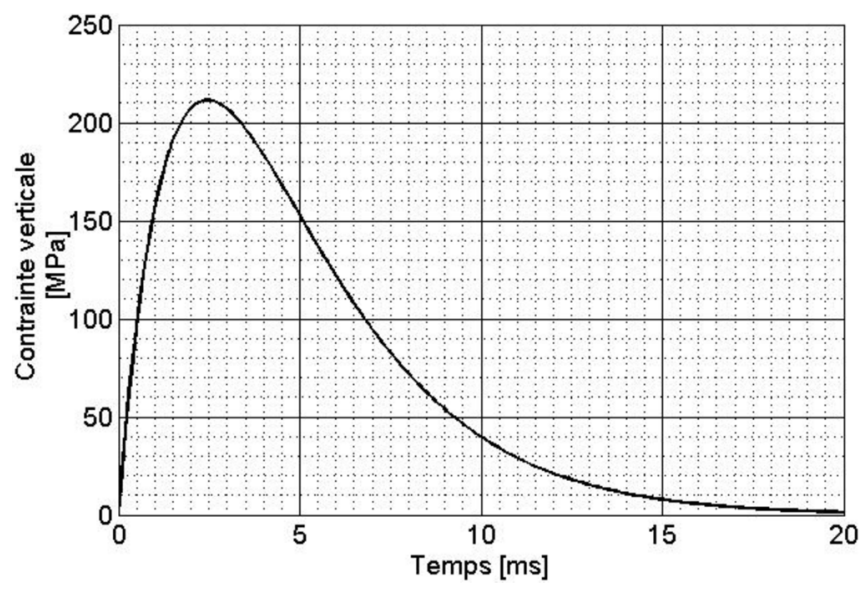

Fig. 5. Courbe de chargement sous un seul coup de marteau pour le pieu semi-infini libre.

Fig. 5. Single hammer blow loading curve for semi-infinite free pile.

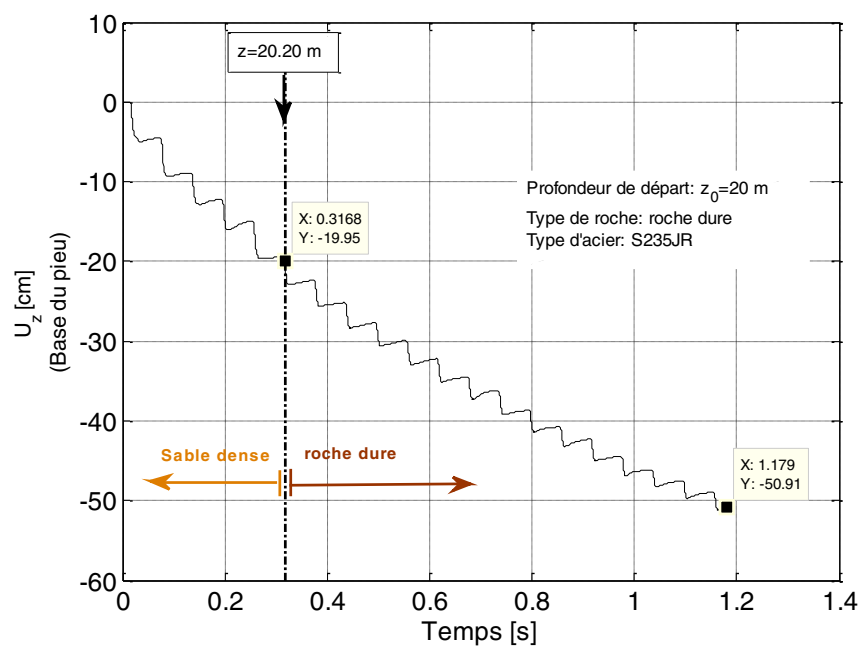

Fig. 6. Histoire de la pénétration du pieu sous 20 coups dans une roche de très bonne qualité (Hamdi, 2016).

Fig. 6. Pile penetration history under 20 blows in a very good quality rock (Hamdi, 2016).

structurale. Les figure $7 \mathrm{a}, \mathrm{b}$, et c montrent la variation des contraintes axiale et de Von Mises en pointe sous, respectivement, les coups $\mathrm{n}^{\mathrm{o}} 1,10$, et 20

Les figure $7 \mathrm{a}, \mathrm{b}$ et $\mathrm{c}$ montrent clairement que les contraintes augmentent dans l'acier en pointe de tube avec la pénétration dans la couche rocheuse : on observe que la 


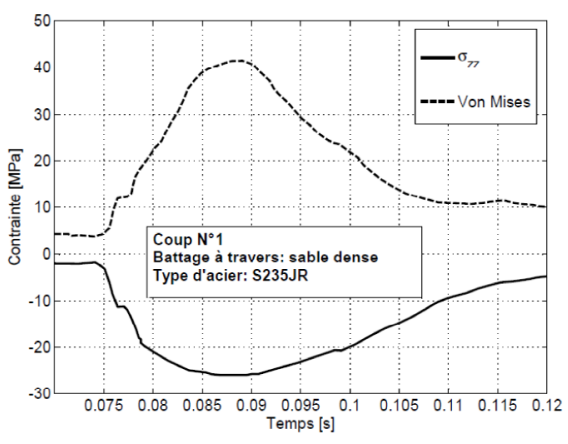

(a)

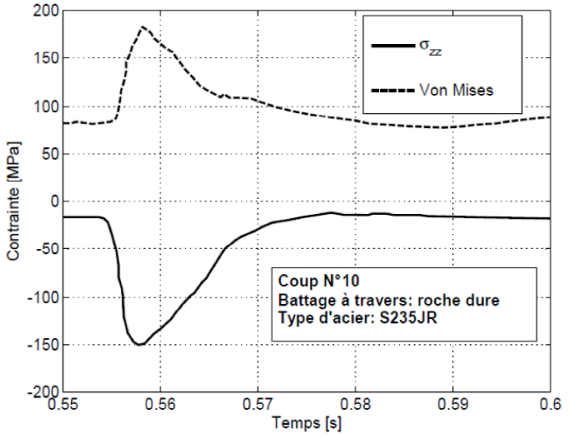

(b)

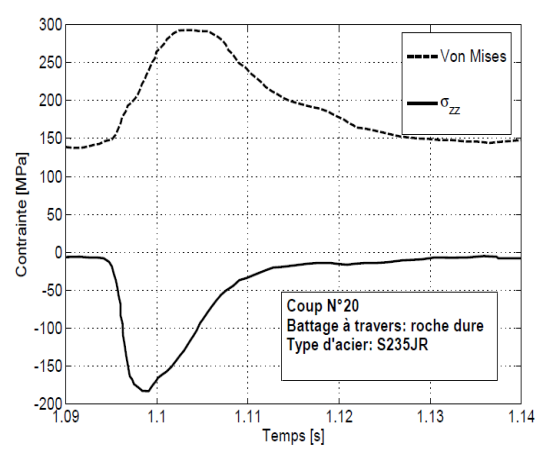

(c)

Fig. 7. Contraintes en pointe de tube: (a) coup $n^{\circ} 1$ (sable dense); (b) coup $n^{\circ} 10\left(10 \mathrm{~cm}\right.$ dans la roche); (c) coup $\mathrm{n}^{\circ} 20$ (30 $\mathrm{cm}$ dans la roche) (Hamdi, 2016).

Fig. 7. Toe stresses: (a) Blow No.1 (dense sand); (b) Blow No.10 (10 cm into rock); (c) Blow No.20 (30 cm into rock) (Hamdi, 2016).

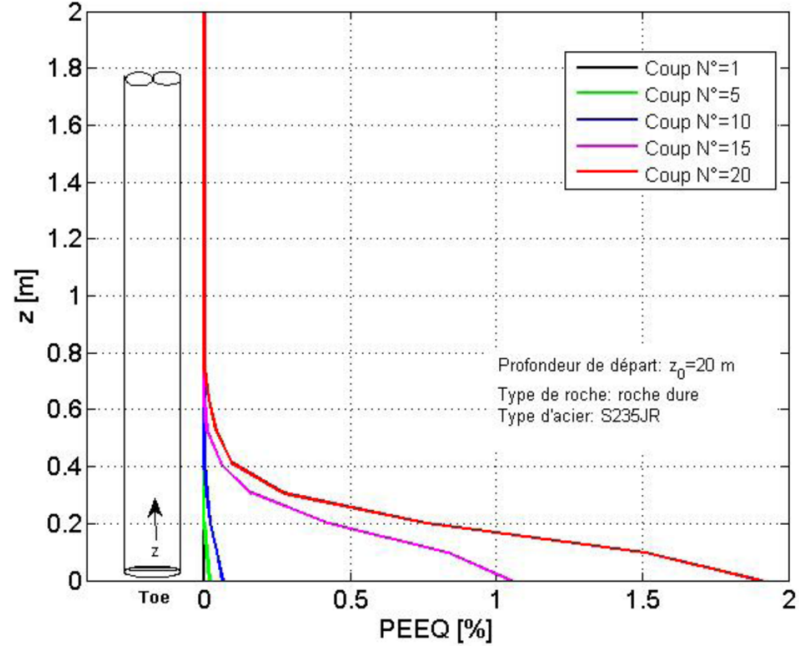

Fig. 8. Évolution de la déformation plastique équivalente à la pointe du pieu (Hamdi, 2016).

Fig. 8. Equivalent plastic strain evolution at pile toe (Hamdi, 2016).

valeur maximale de la contrainte axiale en pointe vaut $5,4 \sigma_{c}$ à une fiche équivalente à 2 fois l'épaisseur du tube pour atteindre $6,6 \sigma_{c}$ à une pénétration équivalente à 6 fois l'épaisseur du tube. Ces valeurs semblent a priori supérieures à la suggestion de 3,0 $\sigma_{c}$ émise par Puech et al. (1990) sur base d'essais de battage en vraie grandeur dans le golfe de Guinée. Ceci peut s'expliquer par la faiblesse de l'épaisseur des bancs de grès pénétrés par ces derniers essais dans le golfe de Guinée. On note par ailleurs l'émergence d'une contrainte de Von Mises résiduelle dans le tube, suite à l'augmentation des contraintes radiales dans le sol autour du tube refoulant le sable et la roche.

La mise en plasticité du tube en acier peut être quantifiée par la déformation plastique équivalente $\left(P E E Q=\sqrt{\frac{2}{3} \varepsilon_{i j}^{p}: \varepsilon_{i j}^{p}}\right)$ définie au départ du tenseur $\varepsilon_{i j}^{p}$ des déformations plastiques. La figure 8 représente l'évolution de la déformation plastique équivalente dans la zone proche de la pointe du pieu pour un acier de qualité courante (S235JR). Après 20 coups de marteau, l'acier qui a pénétré dans la roche de $30 \mathrm{~cm}$, montre des signes non négligeables de plastification sur une longueur de l'ordre du mètre au-dessus de la pointe du pieu.

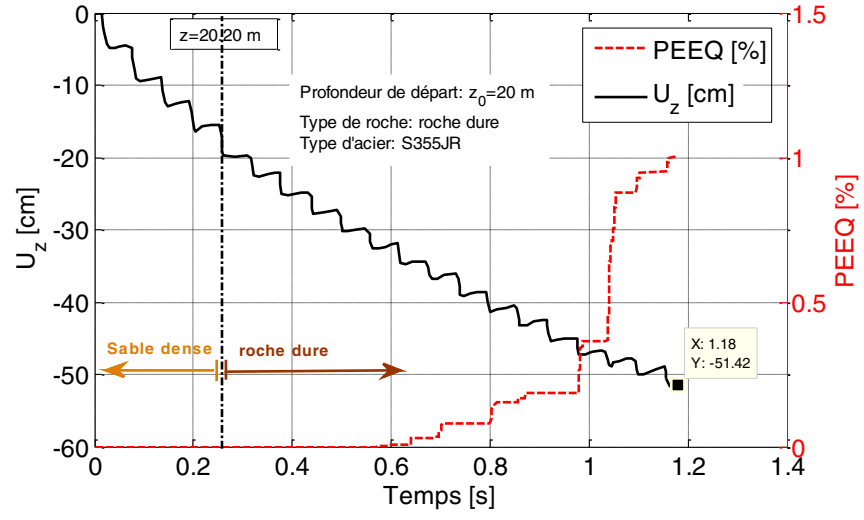

Fig. 9. Déformation plastique équivalente dans l'acier S355JR à la pointe du pieu après 20 coups de marteau (Hamdi, 2016).

Fig. 9. Equivalent plastic strain in S355JR steel at pile toe after 20 hammer blows (Hamdi, 2016).

Afin d'évaluer l'avantage à utiliser un acier de qualité supérieure pour constituer le pieu tubulaire, des simulations numériques mettant en jeu de l'acier S355JR (EN 10025-2) ont été effectuées pour le même cas d'une masse rocheuse de très bonne qualité. La figure 9 présente en fonction du temps non seulement la pénétration du pieu, mais également la variation de la déformation plastique équivalente (PEEQ) en sa pointe. Cette simulation indique que l'acier S355JR est capable de pénétrer les premiers 15 centimètres de roche, soit 3 fois son épaisseur, sans pratiquement entrer en plasticité.

\section{Approche expérimentale}

\subsection{Dispositif de laboratoire}

Le second volet de cet article présente un modèle de laboratoire à échelle réduite permettant la mesure des contraintes transmises lors de battage tout en tenant compte des caractéristiques des matériaux en présence (acier et roche). La recherche FONDEOLE financée par la Région Wallonne (Convention $\mathrm{n}^{\mathrm{O}} 1250394$ de subvention pour une recherche industrielle d'intérêt général) a permis à l'UCL de mettre au point et de valider un modèle de laboratoire pour approcher 


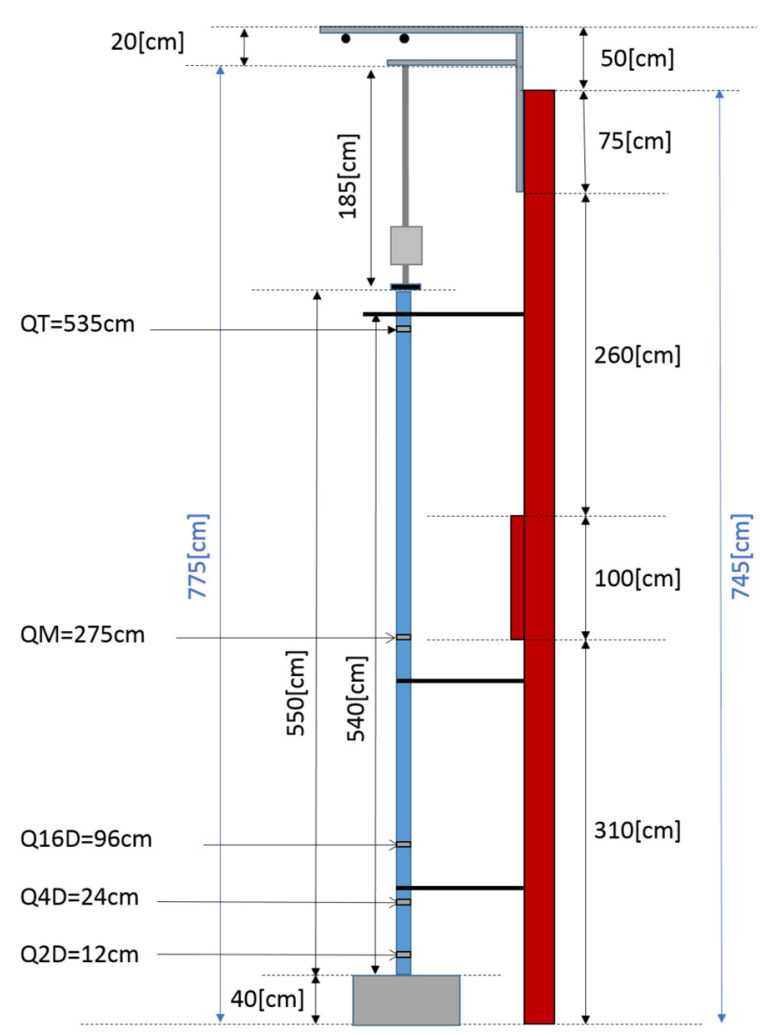

Fig. 10. Schéma général du dispositif de battage pour le pieu de $5,5 \mathrm{~m}$ de long (UCL, 2016).

Fig. 10. General sketch of the driving system for the $5.5 \mathrm{~m}$ long pile (UCL, 2016).

expérimentalement la faisabilité de l'installation de pieux tubulaires dans des massifs indurés tout en évitant leur endommagement.

Le dispositif de battage à échelle réduite conçu en laboratoire se compose d'un pieu en acier inoxydable, de monolithes de roche synthétique et d'un mouton de $10 \mathrm{~kg}$ (Fig. 10). En choisissant un diamètre de tube de l'ordre de $60 \mathrm{~mm}$, le facteur d'échelle est de 30 pour un pieu prototype de l'ordre de $1,8 \mathrm{~m}$ de diamètre tel que typiquement utilisé pour une fondation de type «jacket». Le facteur d'échelle peut monter jusque 150 pour un mono-pieu prototype de $9 \mathrm{~m}$ de diamètre. Les lois de similitude explicitées par Alves et al. (2008) pour l'équation d'onde appliquée au battage des pieux ont été observées.

Le casque est un élément déterminant du système de battage : c'est par celui-ci que l'effort de battage généré par la chute de la masse se transmet au pieu. La qualité des ondes transmises dépend directement de la qualité du casque mis en œuvre. Le schéma du dispositif de battage comprenant le casque mis au point est présenté sur la figure 11. Ce casque en acier favorise une distribution axisymétrique et uniforme des contraintes dans le pieu lors de l'impact. Une butée réglable contrôle la hauteur de chute.

Lors des essais de battage, la mesure de la pénétration du pieu suite à chaque coup ou à une série de coups a été réalisée au moyen d'un appareil de nivellement digital. Le dispositif est composé d'une optique digitale (Fig. 12a) visant une règle à code barre solidarisée sur le pieu modèle (Fig. 12b). La mesure

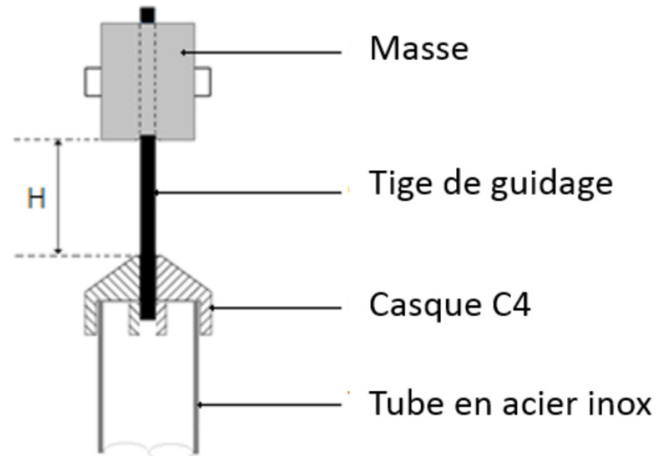

Fig. 11. Dispositif de battage (UCL, 2016).

Fig. 11. Driving assembly (UCL, 2016).

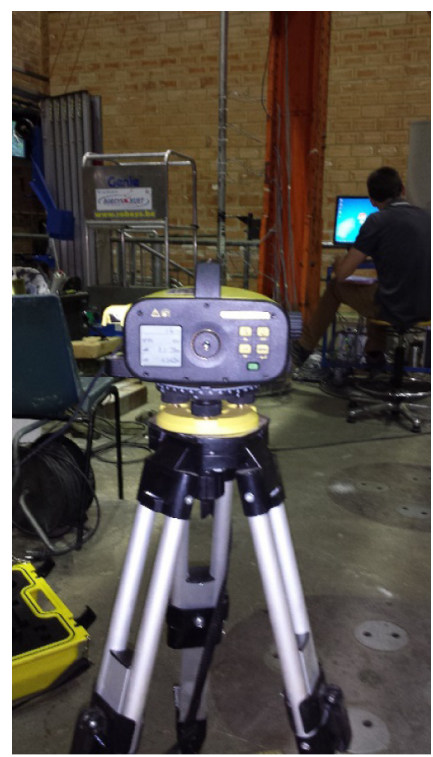

(a)

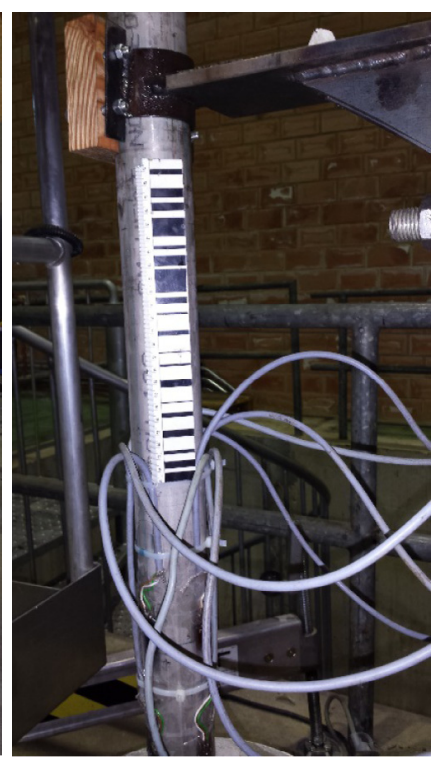

(b)
Fig. 12. Niveau digital : (a) vue de face ; (b) règle graduée (UCL, 2016). Fig. 12. Digital survey: (a) Front view ; (b) Graduated ruler (UCL, 2016).

réalisée avec une résolution de $0,01 \mathrm{~mm}$ assure une précision en pratique de $0,05 \mathrm{~mm}$.

Les matériaux utilisés pour la conception du dispositif de battage à échelle réduite comprennent le matériau permettant la simulation du pieu et les matériaux représentatifs du comportement mécanique de la roche, ainsi que décrit dans les sections qui suivent.

\subsection{Pieux modèles}

Les tubes modèles sont constitués d'acier inox $304 \mathrm{~L}$ dont la limite élastique est définie à la fois par une valeur minimale de $200 \mathrm{MPa}$ et par une valeur moyenne de $265 \mathrm{MPa}$. Ceci procure un meilleur contrôle sur le seuil de plastification du matériau «tube» au cours des essais de battage. Les caractéristiques mécaniques des tubes longitudinalement soudés sélectionnés pour les essais de battage sont synthétisées dans le tableau 2. 
Tableau 2. Tubes et caractéristiques mécaniques acier inox $304 \mathrm{~L}$.

Table 2. Tubes and mechanical properties of stainless steel $304 \mathrm{~L}$.

\begin{tabular}{llllllll}
\hline Tube & $\begin{array}{l}\text { Longueur } \\
\mathrm{m}\end{array}$ & $\begin{array}{l}\text { Diamètre }(\mathrm{D}) \\
{[\mathrm{mm}]}\end{array}$ & $\begin{array}{l}\text { Épaisseur }(\mathrm{t}) \\
{[\mathrm{mm}]}\end{array}$ & $\mathrm{D} / \mathrm{t}$ & \multicolumn{2}{c}{$\begin{array}{c}\text { Limite élastique } \\
\sigma_{\mathrm{Y}}[\mathrm{MPa}]\end{array}$} & \multicolumn{2}{c}{\begin{tabular}{c} 
Contrainte de rupture \\
\cline { 4 - 6 }
\end{tabular}} \\
\hline Court & 2,8 & 60,3 & 1,60 & 38 & $195-239$ & $230-317$ & $500-740$ \\
Mince & 5,5 & 60,3 & 1,60 & 38 & $195-239$ & $230-317$ & $500-740$ \\
Épais & 4,0 & 60,3 & 2,76 & 22 & $205-281$ & N.D. & $515-610$ \\
\hline
\end{tabular}

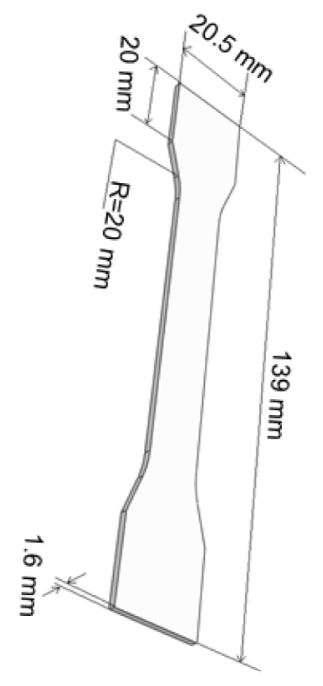

(a)

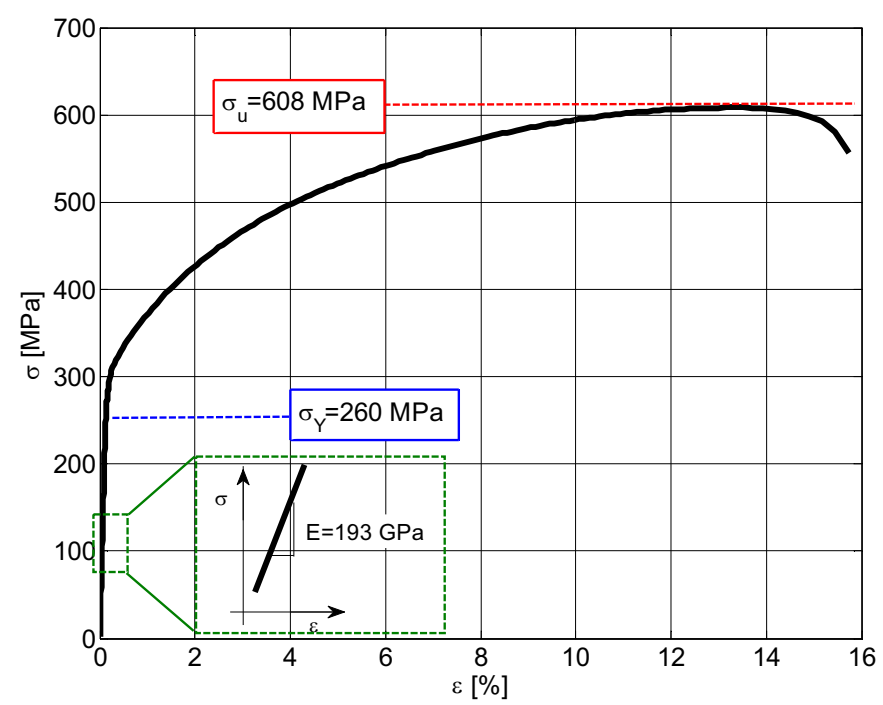

(b)

Fig. 13. (a) Échantillon d'acier; (b) Courbe contrainte - déformation unitaire (UCL, 2016).

Fig. 13. (a) Steel coupon; (b) Stress-strain curve (UCL, 2016).

Afin de définir plus précisément les propriétés mécaniques de l'acier $304 \mathrm{~L}$, des essais de traction ont été réalisés en laboratoire sur des éprouvettes (Fig. 13a) façonnées à partir des tubes utilisés. La figure 13b montre la courbe de contraintedéformation de l'acier du tube mince. On y détecte une limite élastique à $0,2 \% \sigma_{\mathrm{Y}}$ de $260 \mathrm{MPa}$ et une contrainte à la rupture de $608 \mathrm{MPa}$.

Afin de réduire les phénomènes de plastification précoce de la tête du pieu dus à la superposition d'ondes montantes et descendantes, il est préférable de travailler avec un modèle de pieu suffisamment long. Alors qu'une longueur de 2,8 m s'est avérée trop courte à cet égard, une longueur de $5,5 \mathrm{~m}$ s'est révélée être un bon compromis entre l'objectif de limiter la superposition d'ondes et l'encombrement pratique du dispositif. La figure 10 présente le dispositif de battage du pieu modèle de 5,5 $\mathrm{m}$ de long.

\subsection{Instrumentation}

L'instrumentation de ce pieu comprend des jauges longitudinales qui peuvent être montées tantôt en quart de pont (« $\mathrm{Q} »$ pour « quarter bridge »), tantôt en pont complet au moyen de connecteurs adaptés. Chaque niveau de mesure est constitué de quatre jauges placées sur des génératrices extérieurs du tube disposées tous les $90^{\circ}$ (Fig. 14).

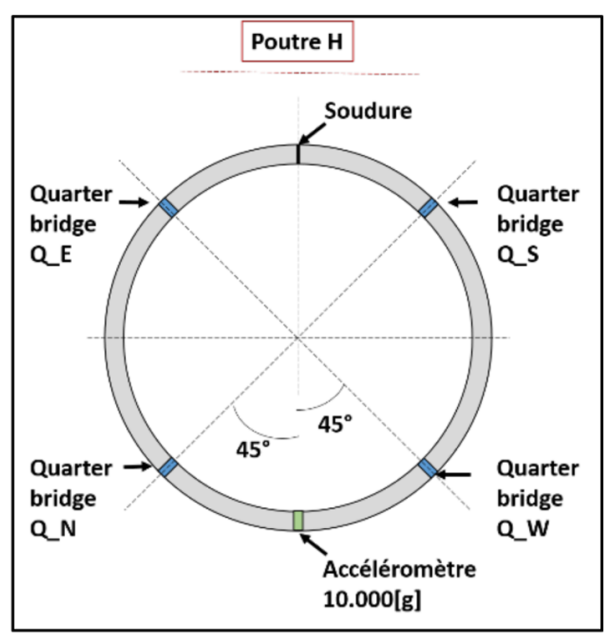

Fig. 14. Coupe de l'instrumentation du pieu de 5,5 $\mathrm{m}$ de long (UCL, 2016).

Fig. 14. Cross-section of the instrumentation for the $5.5 \mathrm{~m}$ long pile (UCL, 2016).

En outre, le niveau d'instrumentation en tête du pieu est doté d'un accéléromètre de gamme $10000 \mathrm{~g}$. Cette gamme élevée de mesure est exigée par la petitesse des éléments 
Tableau 3. Inventaire et nomenclature de l'instrumentation du pieu de $5,5 \mathrm{~m}$ de long.

Table 3. Inventory and nomenclature of the instrumentation for the $5.5 \mathrm{~m}$ long pile.

\begin{tabular}{llr}
\hline $\begin{array}{l}\text { Zone du } \\
\text { pieu } \\
\text { modèle }\end{array}$ & $\begin{array}{l}\text { Nomenclature des jauges de déformation } \\
\text { axiale }\end{array}$ & $\begin{array}{l}\text { Distance } \\
\text { depuis la } \\
\text { tête de } \\
\text { pieu }\end{array}$ \\
\hline Tête & QT_N, QT_S, QT_E, QT_W Accéléromètre & $15 \mathrm{~cm}$ \\
Milieu & QM_N, QM_S, QM_E, QM_W & $275 \mathrm{~cm}$ \\
Pointe & Q16D_N, Q16D_S, Q16D_E, Q16D_W & $454 \mathrm{~cm}$ \\
& Q4D_N, Q4D_S, Q4D_E, Q4D_W & $526 \mathrm{~cm}$ \\
& Q2D_N, Q2D_S, Q2D_E, Q2D_W & $538 \mathrm{~cm}$ \\
\hline
\end{tabular}

métalliques intervenant dans un choc acier-acier, c'est-à-dire en l'absence d'un composant amortissant l'impact entre le marteau et le casque coiffant le pieu. La mesure de la vitesse en tête du pieu s'effectue par intégration dans le temps de l'accélération acquise à une fréquence d'échantillonnage adéquate.

La nomenclature de l'instrumentation équipant le pieu de $5,5 \mathrm{~m}$ de long est récapitulée dans le tableau 3. La position de chaque niveau d'instrumentation est indiquée par sa distance comptée en centimètres depuis la tête du pieu tandis que la position azimutale de chaque jauge est dénotée par une lettre d'un point cardinal ( $E, S, W$ et $N)$. L'éloignement d'un niveau de mesure depuis la pointe du pieu se compte par ailleurs en diamètres de tube (2D, 4D et $16 \mathrm{D})$. Des configurations analogues ont été adoptées pour les pieux de 2,8 et de 4,0 m de longueur, respectant la distinction du niveau de tête (QT) et du milieu (QM) des niveaux proches de la pointe (2D, 4D, etc.).

Les jauges de déformation et l'accéléromètre permettent de suivre la propagation des ondes dans le pieu modèle au cours de son battage. Une chaîne d'acquisition est déployée en laboratoire pour transformer les ondes mécaniques mesurées par les instruments en signaux numériques permettant un traitement par ordinateur. Le système d'acquisition (NI Instruments) se compose de conditionneurs de signaux (Modules SCXI), d'une carte d'acquisition (DAQ-Mx) et d'une interface utilisateur développée à l'UCL (Aquimem-Mx).

La carte d'acquisition offre une fréquence d'échantillonnage maximale globale de $330 \mathrm{kHz}$. La fréquence d'acquisition de chaque canal de mesure s'obtient en divisant ce chiffre par le nombre de canaux simultanément échantillonnés.

\subsection{Validation des mesures dynamiques}

La qualité de la mesure de l'accélération peut être vérifiée par sa confrontation avec les mesures de déformation en tête de pieu : l'intégration de l'accélération permet d'obtenir la vitesse du pieu $v_{\text {рieu}}$. Le rapport de la vitesse en tête de pieu sur la célérité des ondes mécaniques dans l'acier inoxydable $(c=5031 \mathrm{~m} / \mathrm{s})$ représente une quantité physique qui s'apparente au nombre de Mach en aérodynamique. Comme elle peut être vue comme une déformation unitaire ${ }_{c i n}=\frac{v_{\text {pieu }}}{c}$, nous nous permettrons de la dénommer «déformation cinétique» et l'exprimerons en microdéformations ( $\mu$ def ou $\mu$ str) afin de la comparer à la déformation mécanique mesurée directement par les jauges de déformation. La figure 15 montre la comparaison entre les deux déformations mécanique et cinétique pour un essai effectué sous une hauteur de chute de $20 \mathrm{~cm}$ sur le pieu de $5,5 \mathrm{~m}$ de long reposant sur un monolithe de très faible résistance.

Nous observons sur cette figure que la correspondance est bien vérifiée jusqu'au moment de l'écho au-delà duquel les signaux divergent en réaction à la faible résistance à la pointe. Lorsque l'on multiplie ces deux signaux par le module d'Young de l'acier, on vérifie la relation d'impédance spécifique $(\sigma=v \sqrt{\rho E})$ déduite de l'équation d'onde.

Compte tenu du module d'élasticité adopté de $200 \mathrm{GPa}$, on peut facilement convertir une déformation mécanique unitaire de $100 \mu$ str en une contrainte de $20 \mathrm{MPa}$, c'est-à-dire une force de $5,9 \mathrm{kN}$ sur les $295 \mathrm{~mm}^{2}$ que représente la section $A$ d'acier du pieu modèle de $1,6 \mathrm{~mm}$ d'épaisseur. On peut également convertir une déformation cinétique de $100 \mu$ str en une vitesse de $0,5 \mathrm{~m} / \mathrm{s}$.

\section{Matériaux des monolithes}

Pour simuler la présence de la roche, des monolithes en roche synthétique ont été utilisés en laboratoire afin d'atteindre une gamme intéressante du rapport de résistance acier/roche $\left(\sigma_{Y} / \sigma_{c}\right)$ allant de 7 à 50 tout en s'affranchissant de la dispersion élevée des roches naturelles. Notons que ce contraste est défini par le rapport de la limite élastique en extension de l'acier sur la résistance à la compression simple de la roche. Comme la limite élastique de l'acier $\sigma_{Y}$ est de $265 \mathrm{MPa}$, les monolithes à confectionner en laboratoire devaient donc présenter des résistances à la compression simple $\sigma_{\mathrm{c}}$ couvrant la gamme $\sigma_{c}=4-38[\mathrm{MPa}]$. Par référence à la classification usuelle des roches, cette gamme couvre des résistances de roche qualifiées de «très faibles » $\left(0,5 \mathrm{MPa}<\sigma_{\mathrm{c}}<6 \mathrm{MPa}\right)$, « faibles » $(6 \mathrm{MPa}$ $\left.<\sigma_{\mathrm{c}}<20 \mathrm{MPa}\right)$ et «moyennes » $\left(20 \mathrm{MPa}<\sigma_{\mathrm{c}}<50 \mathrm{MPa}\right)$.

\subsection{Monolithes de très faible résistance $\left(0,5 \mathrm{MPa}<\sigma_{\mathrm{c}}<6 \mathrm{MPa}\right)$}

Les monolithes retenus pour simuler la présence d'une masse rocheuse de faible résistance sont des monolithes en mortier, improprement appelé «béton cellulaire » d'une masse volumique sèche de l'ordre de $650 \mathrm{~kg} / \mathrm{m}^{3}$. Leur porosité dépasse généralement $60 \%$. Ces monolithes fabriqués en usine pour l'industrie de la construction ont été caractérisés en laboratoire : ils possèdent une résistance à la compression de 4,2 $\mathrm{MPa}$ et à la traction de $0,6 \mathrm{MPa}$. Leurs modules de Young et coefficient de Poisson sont respectivement de $1400 \mathrm{MPa}$ et de 0,23 .

\subsection{Monolithes de faible ( $\left.6 \mathrm{MPa}<\sigma_{\mathrm{c}}<20 \mathrm{MPa}\right)$ et moyenne (50 $\left.\mathrm{MPa}<\sigma_{\mathrm{c}}<50 \mathrm{MPa}\right)$ résistances}

La gamme de résistance à la compression simple des monolithes en mortier employés pour simuler la présence de couches de roche de faible et moyenne résistance devait se situer entre 8 et $30 \mathrm{MPa}$. Cette gamme de résistance n'étant pas facilement disponible dans les matériaux du commerce, il a été choisi de fabriquer en laboratoire des monolithes avec du mortier de qualité ajustée. 


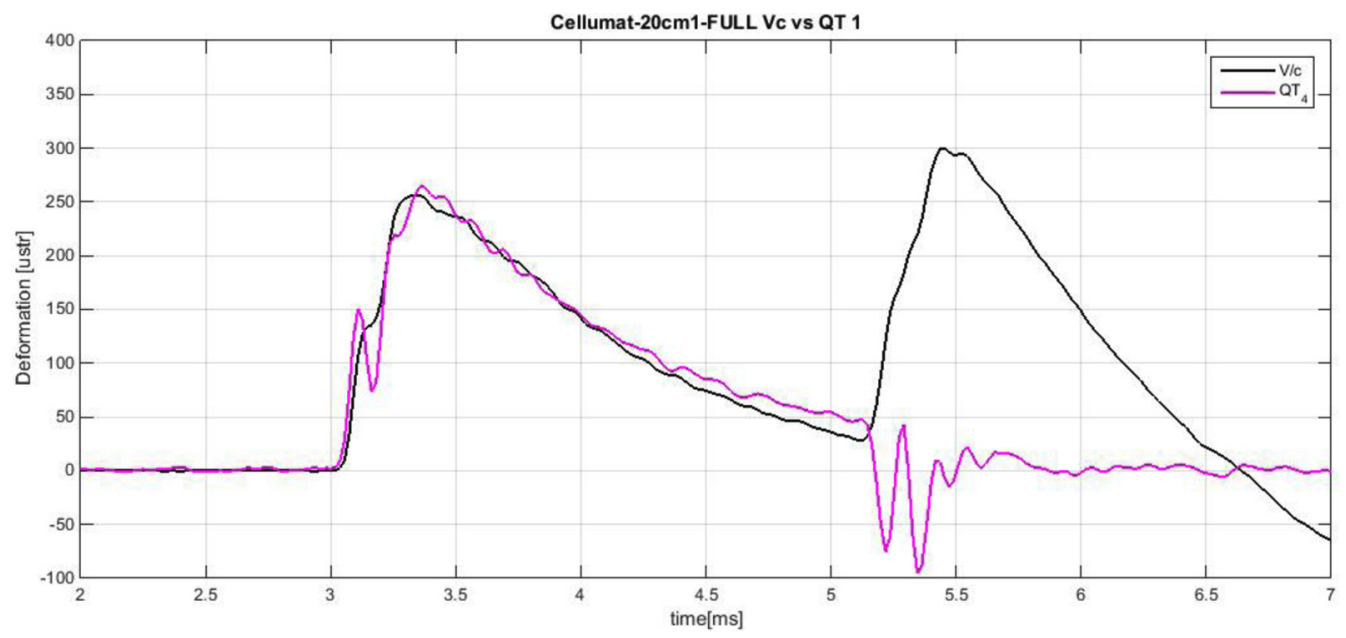

Fig. 15. Comparaison entre les déformations cinématique (v/c) et mécanique ( $\varepsilon$ ) (UCL, 2016).

Fig. 15. Comparison between kinematic (v/c) and mechanical (E) strains (UCL, 2016).

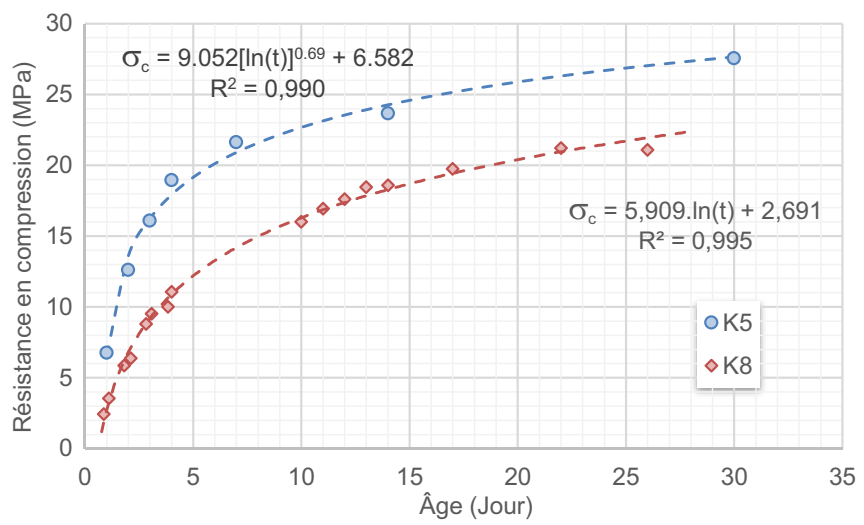

Fig. 16. Évolution de la résistance en compression des mélanges K5 et K8 (Victor, 2017).

Fig. 16. Evolution of the UCS of the K5 and K8 mixes (Victor, 2017).

Un mortier à granulométrie fine a été mélangé à de la kaolinite dans le but d'obtenir la résistance à la compression cible tout en évitant la présence de points durs à la base du tube présentant une épaisseur de seulement 1,6 $\mathrm{mm}$. La granulométrie fine du mortier employé à l'état pur (sans kaolinite) entraîne toutefois une augmentation rapide de sa résistance lors des premiers jours de cure, pour atteindre $65 \mathrm{MPa}$ à 28 jours, et ce, pour une teneur en eau de $18 \%$. Cette résistance a dû être abaissée par incorporation de kaolinite au mélange. Entre autres, deux mélanges ont été retenus pour les expériences en laboratoire. Ils sont dénommés $\mathrm{K} 5$ et $\mathrm{K} 8$ selon leurs proportions en kaolinite exprimées en pourcents. La porosité de ces deux mélanges est estimée de l'ordre de 3 et $5 \%$, respectivement.

À 28 jours de cure, le mélange $\mathrm{K} 5$ atteint une résistance à la compression simple de 27,4 MPa, une résistance à la traction par fendage de 1,5 MPa et un module de Young de $21500 \mathrm{MPa}$. L'évolution temporelle de la résistance à la compression du mélange $\mathrm{K} 5$ a été caractérisée sur base d'essais sur éprouvettes prismatiques conformément à la norme EN 196-1 : 1995. La figure 16 montre la variation de la résistance en compression du mélange $\mathrm{K} 5$ en fonction du temps de cure. Chaque point y représente la moyenne des résistances d'au moins quatre éprouvettes écrasées le même jour. Il est à noter que les déviations maximales observées par rapport à la valeur de la résistance moyenne pour chaque jour sont restées inférieures à $10 \%$.

L'évolution temporelle de la résistance moyenne du mélange K8 est également présentée sur la figure 3. Ce deuxième mélange contenant $8 \%$ de kaolinite atteint une résistance à la compression de $\sigma_{c}=10$ à $11 \mathrm{MPa}$ après 4 jours de cure, de $16 \mathrm{MPa}$ après 10 jours de cure, de $18 \mathrm{MPa}$ après 13 jours de cure, et enfin de $22 \mathrm{MPa}$ après 28 jours de cure. Cette évolution de la résistance en fonction du temps de cure est exploitée afin de régler, par un contrôle strict du calendrier des essais, la résistance voulue lors de l'épreuve de battage du tube dans le monolithe.

\section{Essais de battage dans le mortier cellulaire $\left(\sigma_{\mathrm{c}}=4,2 \mathrm{MPa}\right)$}

\subsection{Pieu court}

La séquence de battage du pieu de 2,8 $\mathrm{m}$ de long effectuée dans le mortier cellulaire a généralement compris 5 impacts pour chacune des hauteurs de chutes suivantes : $5,20,5,20,40$, 20 et $40 \mathrm{~cm}$. Les résultats de cette séquence de battage sont présentés sur la figure 17 sous la forme de quatre diagraphies en fonction de la pénétration: (a) la hauteur de chute du mouton $[\mathrm{m}]$ et son énergie potentielle $[\mathrm{J}]$ sachant qu'il possède une masse de $10 \mathrm{~kg}$, (b) le refus ou enfoncement par coup [mm], (c) la déformation maximale mesurée à différents niveaux, et (d) la résistance du tube à la pénétration $[\mathrm{kN}]$. Cette dernière est estimée de deux manières: (1) le produit de la déformation maximale mesurée en pointe (en pratique $2 \mathrm{D}$ audessus) et du module de raideur $E A$ du tube ; (2) par formule de battage selon Hiley (1925), moyennant calibration sur un ensemble de résistances moyennes dégagées pour différentes hauteurs de chute.

Lors de ces tests, 8 canaux de la chaîne d'acquisition ont été échantillonnés à $41 \mathrm{kHz}:$ Q2D_N, Q2D_S, Q4D_N, 
A. Holeyman : Rev. Fr. Geotech. 2017, 153, 1

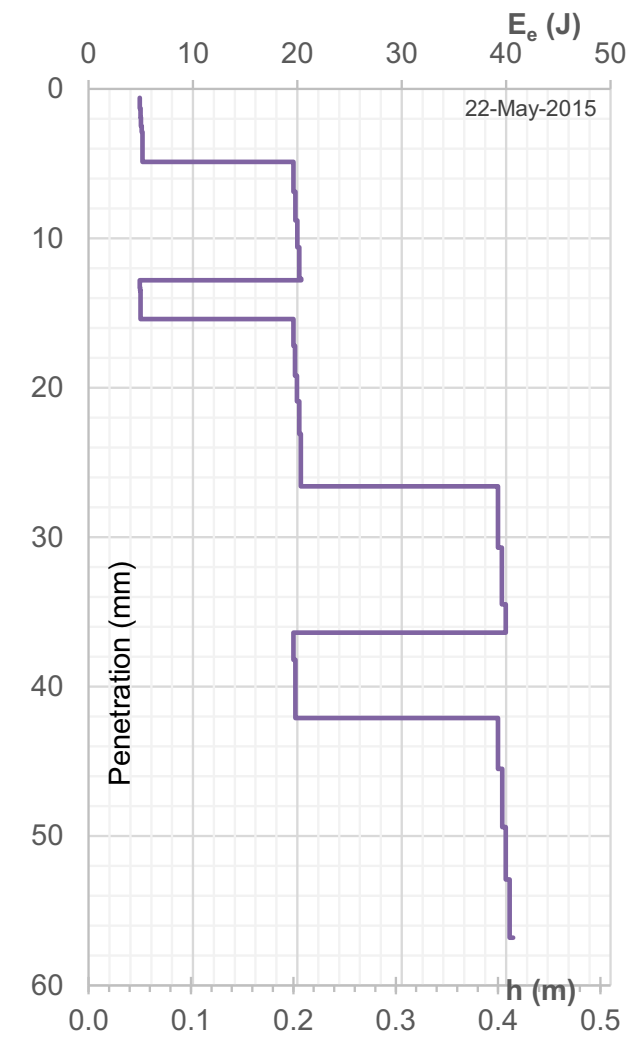

(a)

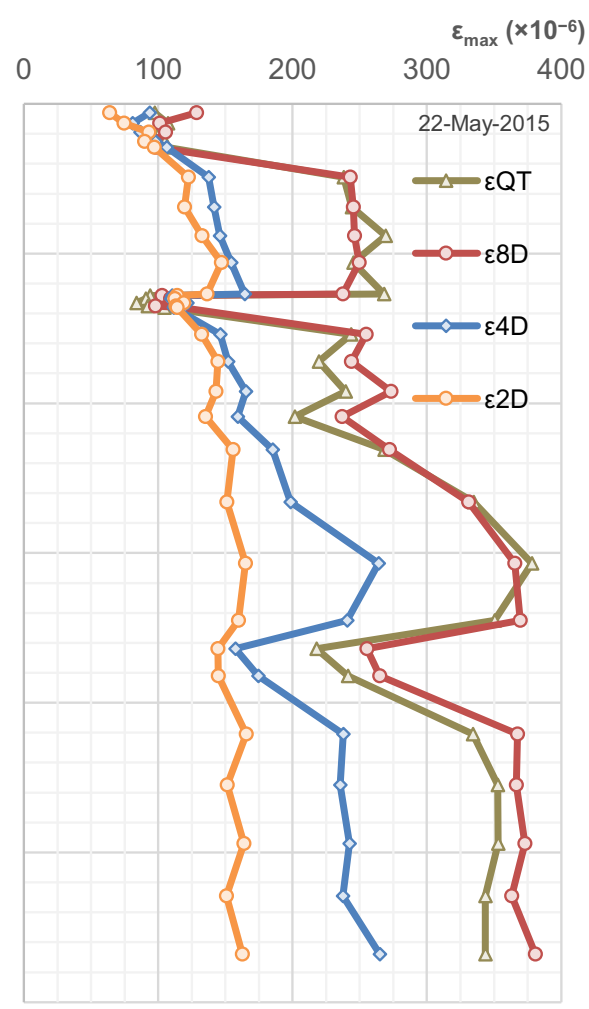

(c)

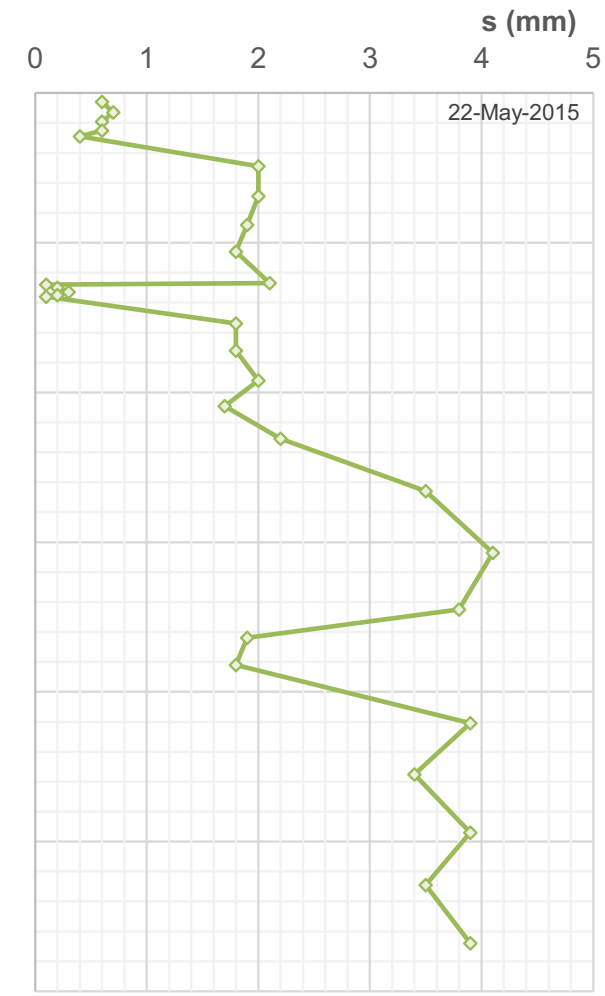

(b)

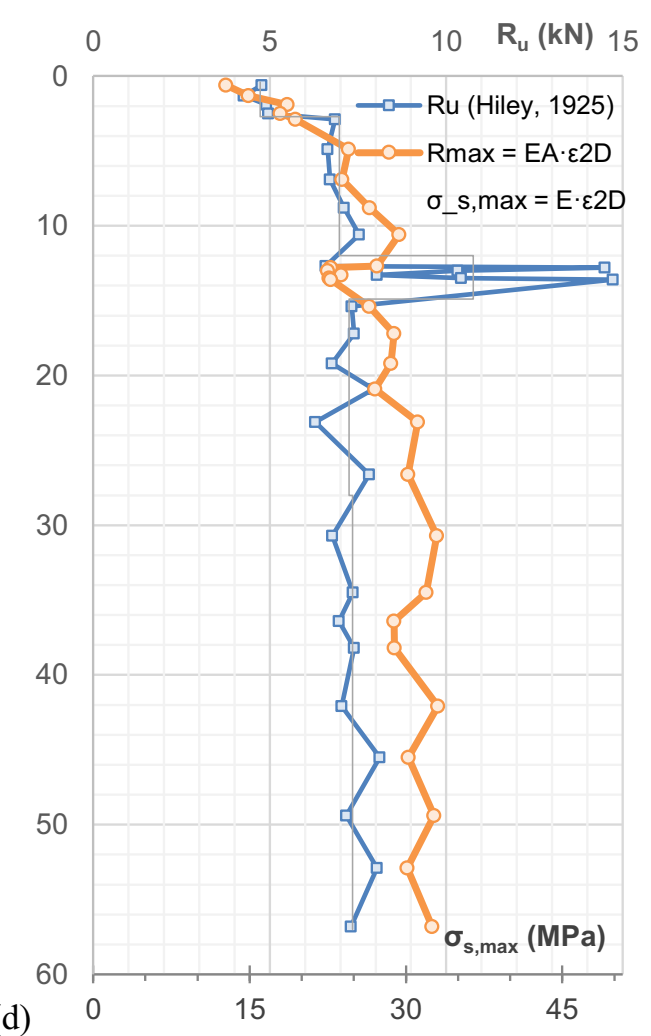

Fig. 17. Pieu court battu dans le mortier cellulaire (a) hauteur de chute/énergie; (b) refus; (c) déformation à différents niveaux; (d) résistance du tube à la pénétration et contrainte maximale en pointe (Victor, 2017).

Fig. 17. Short tube driven into cellular mortar (a) drop height/energy; (b) set; (c) peak strain at different levels; (d) tube penetration resistance and toe peak stress (Victor, 2017). 


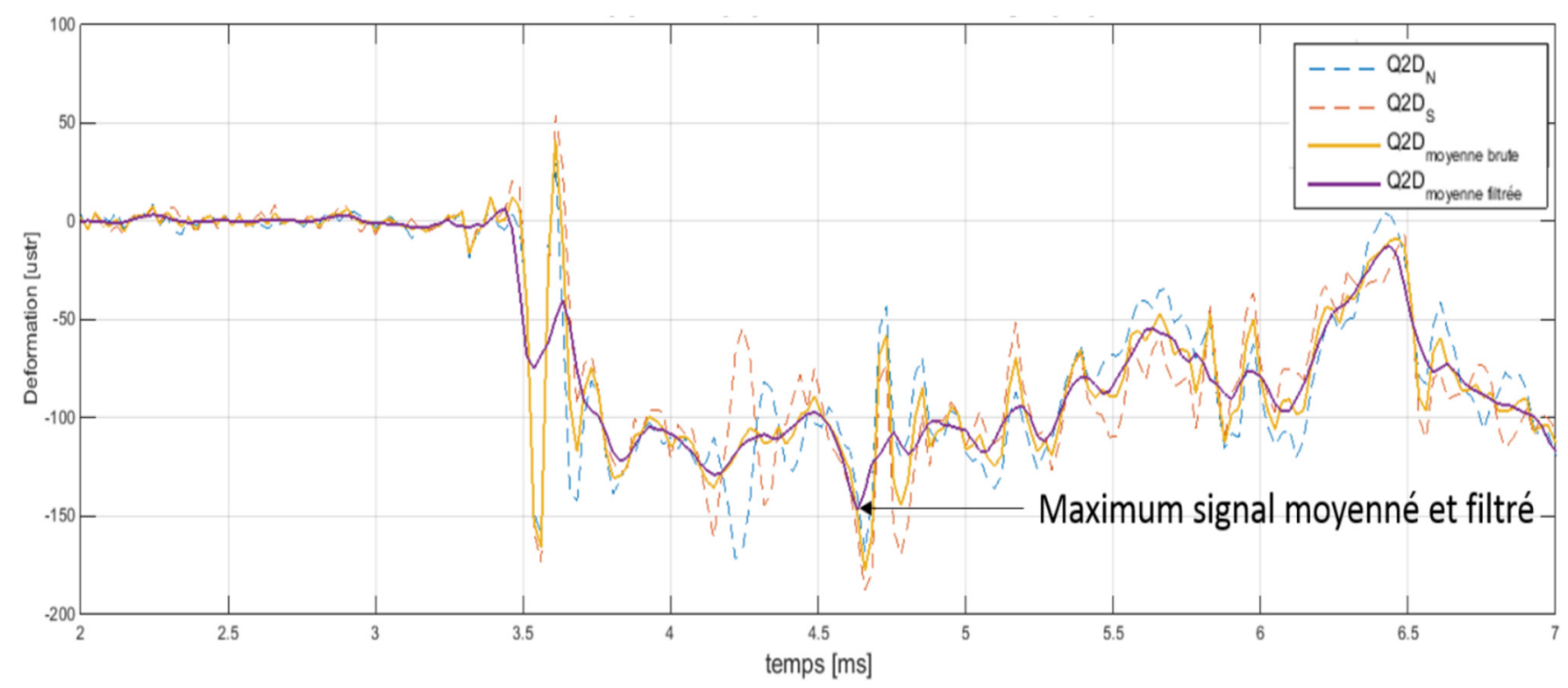

Fig. 18. Signaux de déformation en pointe du pieu court dans le mortier cellulaire sous une chute de 20 cm (UCL, 2016).

Fig. 18. Strain signals at the toe of the short pile driven into cellular mortar under a $20 \mathrm{~cm}$ drop (UCL, 2016).

Q8D_N, Q8D_S, QT_N, QT_E ainsi que l'accéléromètre en tête. Les valeurs de déformation indiquées sur ces graphiques sont calculées en faisant la moyenne numérique des signaux des jauges disponibles à un niveau donné (généralement $\mathrm{N} \&$ S) puis en filtrant le signal obtenu par moyenne glissante sur 5 échantillons $(\Delta t=0,12 \mathrm{~ms})$. Un exemple de signal moyenné et filtré pour le niveau d'instrumentation Q2D sous un impact de $20 \mathrm{~cm}$ est montré sur la figure 18 avec la valeur maximale retenue. Les valeurs indiquées sur la figure 17(a) sont les maxima sur l'intervalle $t=3$ à $5 \mathrm{~ms}$ des signaux filtrés suivants : Q2D (N \& S), Q4D (N), Q8D (N \& S) et QT (S \& W).

On peut constater une assez bonne correspondance entre les valeurs de la résistance du tube à la pénétration obtenues par mesure directe et via la formule de battage, en dépit des observations suivantes: (1) on ne dispose que de 2 jauges (N\&S) en pointe du tube; (2) la formule de battage a tendance à donner une résistance plus élevée pour une hauteur de chute moindre. On note une augmentation de la résistance du tube à la pénétration avec la profondeur, assez marquée au début, mais qui semble évoluer ensuite vers une valeur asymptotique.

Ainsi qu'exposé plus haut, un tube de 5,5 $\mathrm{m}$ de long a été mis en œuvre afin de permettre à l'onde de compression générée lors de l'impact au niveau de la tête du pieu de s'atténuer avant qu'elle ne se superpose à l'onde réfléchie à la base du tube. Grâce à la longueur de ce tube, plusieurs résistances de roche ont été isolées par instrumentation en pointe afin d'étudier la faisabilité du battage de pieux tubulaires en fonction de la résistance de la couche de roche rencontrée. Deux tubes de grande longueur sont à distinguer : «le tube mince» $(t=1,6 \mathrm{~mm})$ et le tube « épais«» $(t=2,8 \mathrm{~mm})$.

\subsection{Pieu long}

La séquence de battage avec le pieu de 5,5 $\mathrm{m}$ de longueur a généralement compris 4 impacts pour chacune des hauteurs de chutes suivantes : 5, 20,5,20,40, 20 et $40 \mathrm{~cm}$. Les résultats de cette séquence de battage sont présentés en fonction de la pénétration sur la figure 19. Les valeurs indiquées sur ces graphiques sont calculées par moyennage et filtrage comme indiqué plus haut sur les jauges en pont complet suivantes: QT $(\mathrm{N}, \mathrm{E}, \mathrm{S} \& \mathrm{~W}), \mathrm{QM}(\mathrm{N}, \mathrm{E}, \mathrm{S} \& \mathrm{~W})$ et Q2D (N, E, S \& W).

On peut noter ici une excellente correspondance entre les valeurs de la résistance du tube à la pénétration car on dispose de 4 jauges $(\mathrm{N}, \mathrm{E}, \mathrm{S} \& \mathrm{~W})$ en pointe du tube. L'allure caractérisant l'augmentation de la résistance du tube à la pénétration avec la profondeur se confirme.

\subsection{Pieu épais}

Le tube épaissi à $2,77 \mathrm{~mm}$ sur ses 4 mètres inférieurs a été battu au moyen de 5 à 8 impacts pour chacune des hauteurs de chutes suivantes : $5,10,5,20,10$ et $20 \mathrm{~cm}$. Les résultats de cette séquence de battage sont présentés en fonction de la pénétration sur la figure 20. Les valeurs indiquées sur ces graphiques sont calculées par moyennage et filtrage comme indiqué plus haut.

Afin d'étudier l'effet du changement d'épaisseur, les jauges de déformation Q19DR et Q15DR ont été placées de part et d'autre du changement de section. On note que l'augmentation de section induit une réduction des déformations mesurées au niveau de la base du pieu.

\subsection{Conclusion préliminaire}

Les essais de battage des tubes mince et épais dans le mortier cellulaire indiquent qu'il est plutôt aisé de battre un tube en acier dans un matériau fortement poreux dont la résistance à la compression est inférieure à $6 \mathrm{MPa}$. Dans cette catégorie de matériau induré, une hauteur de chute faible $(40 \mathrm{~cm})$ génère un enfoncement du tube de plusieurs millimètres sans que la contrainte en pointe n'excède 20 à 

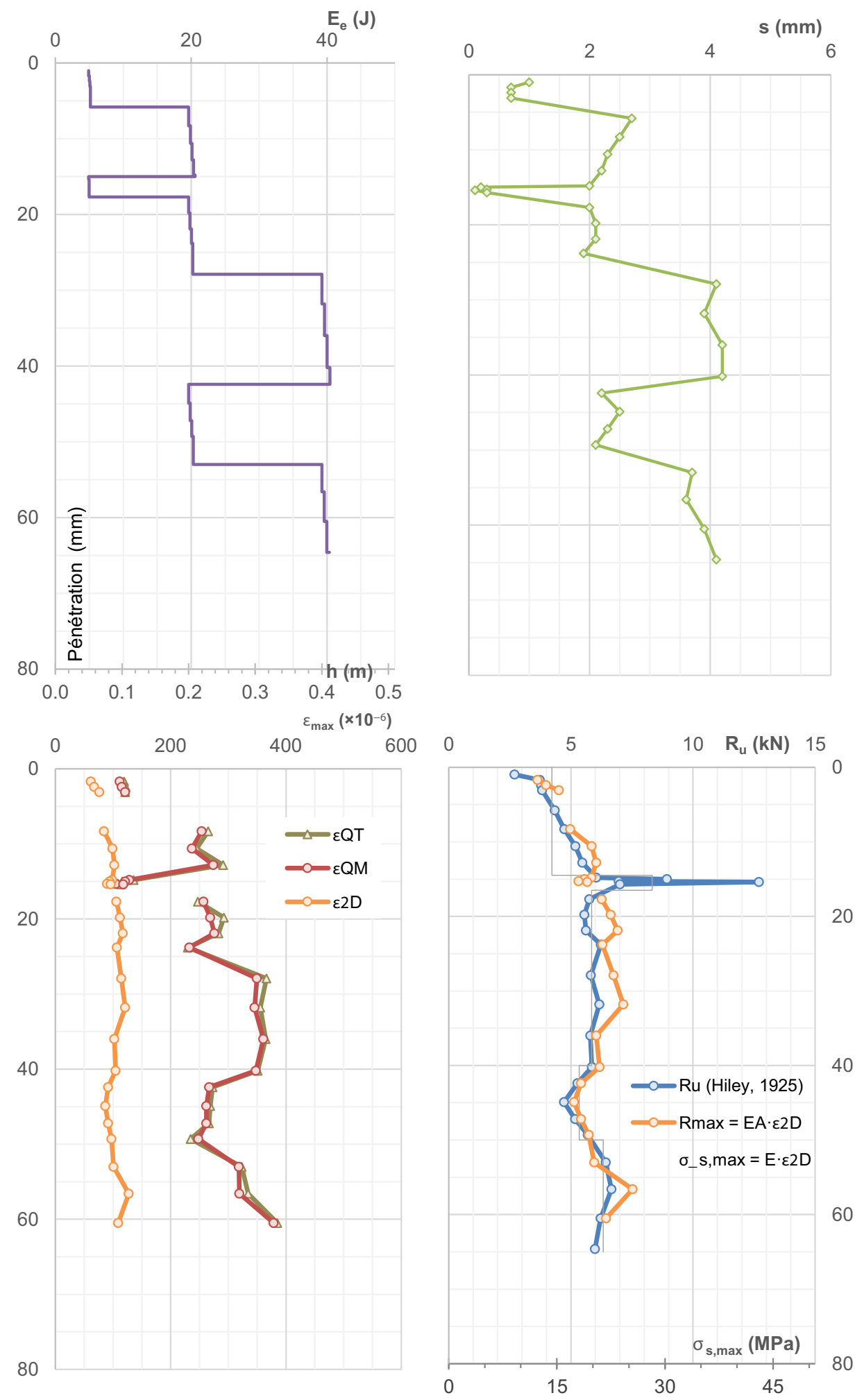

Fig. 19. Pieu long battu dans le mortier cellulaire (a) hauteur de chute/énergie ; (b) refus ; (c) déformation à différents niveaux ; (d) résistance du tube à la pénétration et contrainte maximale en pointe (Victor, 2017).

Fig. 19. Long pile driven in cellular mortar (a) drop height/energy; (b) set; (c) peak strain at different levels; (d) tube penetration resistance and toe peak stress (Victor, 2017). 

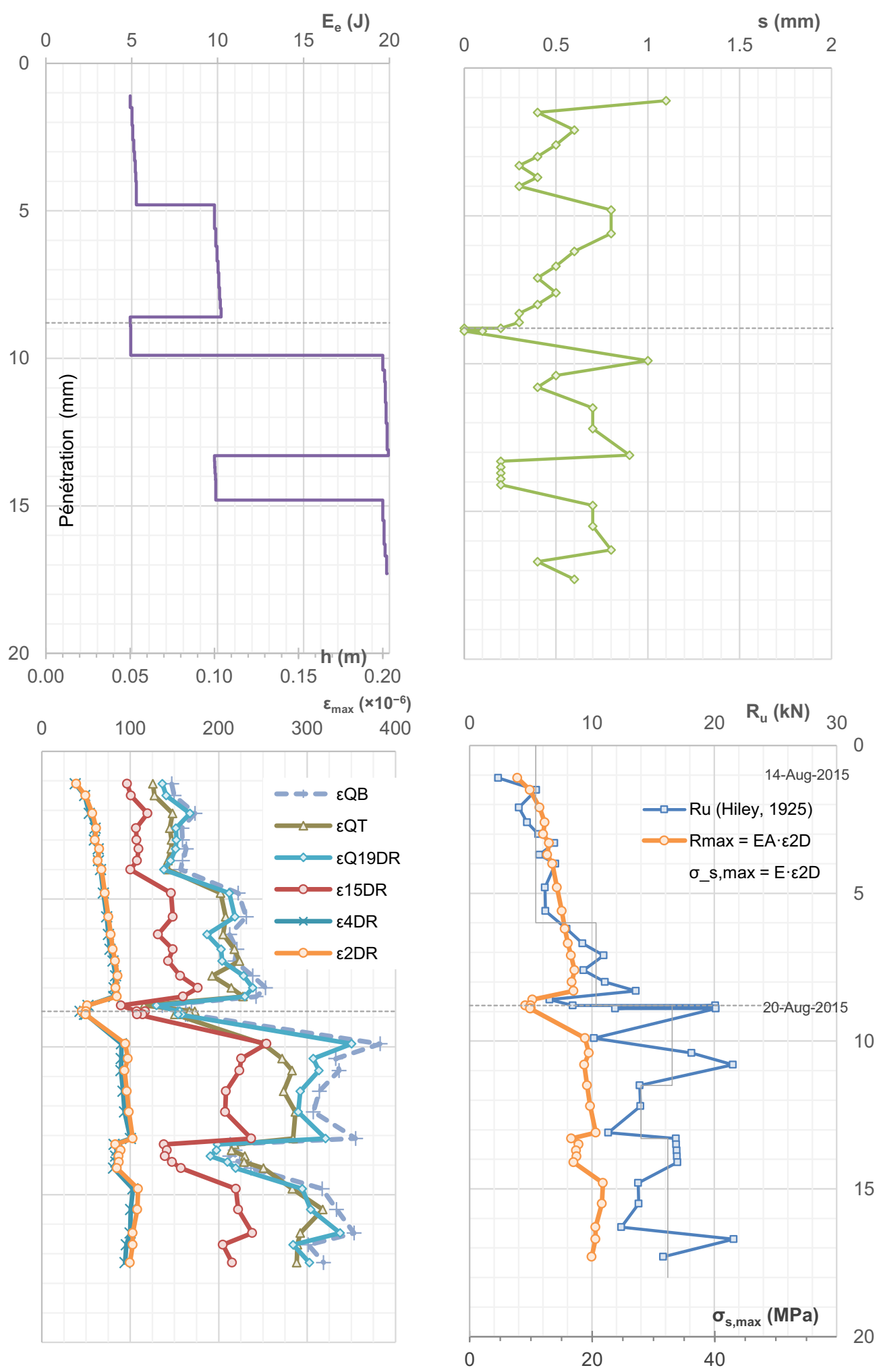

Fig. 20. Pieu épais battu dans le mortier cellulaire (a) hauteur de chute/énergie ; (b) refus ; (c) déformation à différents niveaux ; (d) résistance du tube à la pénétration et contrainte maximale en pointe (Victor, 2017).

Fig. 20. Thick pile driven in cellular mortar (a) drop height/energy; (b) set; (c) peak strain at different levels; (d) tube penetration resistance and toe peak stress (Victor, 2017). 
$25 \mathrm{MPa}$, soit 5 à $6 \sigma_{c}$ pour une vitesse incidente en tête de pieu de l'ordre de $1,8 \mathrm{~m} / \mathrm{s}$.

Cette conclusion débouche sur l'intérêt de réaliser des essais de battage dans des matériaux plus résistants, par exemple le mortier K5 à 28 jours, tel que décrit dans la section qui suit.

\section{Essais de battage dans des mortiers avec bentonite}

\subsection{Essai de battage du pieu de $2,8 \mathrm{~m}$ dans du mortier $\mathrm{K} 5\left(\sigma_{\mathrm{c}}=26,8 \mathrm{MPa}\right)$}

Une séquence de battage a été effectuée dans la roche synthétique $\mathrm{K} 5$ affichant une résistance à la compression de 26,8 MPa. Elle comprenait des séries de 3 à 6 coups faisant varier la hauteur de chute entre 5 et $150 \mathrm{~cm}$. Les résultats de cette séquence de battage sont présentés en fonction de la pénétration sur la figure 21 . Les valeurs indiquées sur ces graphiques sont calculées par moyennage et filtrage comme indiqué plus haut.

La figure 22 montre les signaux de déformation correspondant aux niveaux d'instrumentation Q2D, Q4D, $\mathrm{Q} 8 \mathrm{D}$ et $\mathrm{QT}$ sous le $2^{\mathrm{e}}$ impact de $80 \mathrm{~cm}$ à $37 \mathrm{~mm}$ de pénétration. Nous pouvons observer sur cette figure que l'onde en tête (QT) présente deux pics. Le premier pic correspond au pic de l'onde incidente (onde descendante générée par l'impact de la masse). Le deuxième pic, plus élevé que le premier, correspond à la superposition de l'onde incidente et de l'onde réfléchie. Ce pic est plus élevé car l'onde incidente a pu se réfléchir en pointe de pieu grâce à la réaction disponible à la base. Cette amplitude se confirme sur le signal proche de la pointe du pieu (Q2D) qui affiche un pic filtré de $850 \mu$ str correspondant à une contrainte au contact acier-roche d'environ $170 \mathrm{MPa}$, soit $5,7 \sigma_{c}$ pour une vitesse incidente en tête de pieu de l'ordre de $2,5 \mathrm{~m} / \mathrm{s}$.

Les déformations unitaires correspondant au premier coup de $130 \mathrm{~cm}$ de hauteur de chute (Fig. 23) indiquent que l'amplification de l'onde en tête résultant de la superposition des ondes incidente et réfléchie est plus élevée que dans le cas de la chute de $80 \mathrm{~cm}$ (Fig. 22). L'amplification du pic de déformation en tête s'accroît donc avec la hauteur de chute, ce qui est néfaste pour l'intégrité de la tête du pieu. L'amplitude maximale du signal filtré en pointe du pieu (Q2D) atteint environ $1000 \mu$ str, ce qui correspond à une contrainte supposée élastique au contact acier-roche d'environ $200 \mathrm{MPa}$, soit $7,6 \sigma_{c}$ pour une vitesse estimée en pointe de pieu de l'ordre de $4,2 \mathrm{~m} / \mathrm{s}$.

Les essais réalisés dans le mortier K5 de $\sigma_{c}=26,8 \mathrm{MPa}$ établissent qu'il est pratiquement impossible de faire pénétrer le tube sans excéder sa limite élastique. Pour une profondeur de pénétration supérieure à $25 \mathrm{~mm}$, on note en effet (Fig. 21) que le refus devient inférieur à $1 \mathrm{~mm}$ pour une hauteur de chute de 80 , voire $130 \mathrm{~cm}$ alors que la déformation unitaire de l'acier dépasse sa limite élastique à partir d'une hauteur de chute de $80 \mathrm{~cm}$.

Cet excès est confirmé par la détection de déformations permanentes dans l'acier à partir d'une hauteur de chute de $80 \mathrm{~cm}$. L'apparition de la plastification est établie sur base de l'analyse de l'histoire complète des signaux de déformation. Lorsque le pieu se plastifie sous l'effet d'une contrainte dépassant la limite élastique, les jauges en zone plastifiée présentent après impact une déformation rémanente vis-à-vis de leur état avant impact. L'écart entre les moyennes de déformation après et avant impact permet de caractériser l'évolution de la plastification du tube. L'essai dans le mortier K5 à 28 jours souligne également l'intérêt de réaliser des essais de battage dans un matériau moins résistant, par exemple un mortier K5 plus jeune ou un mortier K8, tel que décrit dans la section qui suit.

\subsection{Essais de battage dans le mortier $\mathrm{K} 8\left(\sigma_{c}=11 \mathrm{MPa}\right)$}

Un essai de battage a été réalisé dans un monolithe de mortier K8 à 4 jours de cure possédant une résistance à la compression de $11 \mathrm{MPa}$. Le pieu mince a été battu sous un minimum de 4 impacts pour chacune des hauteurs de chutes suivantes : 20, 40, 20, 40,80, 40 et $80 \mathrm{~cm}$. Les résultats de cette séquence de battage sont présentés sur la figure 24 . Les valeurs indiquées sur ces graphiques sont calculées de telle sorte que QT, QM, et Q2D sont les déformations mesurées respectivement à la tête du tube $\left(z=\mathrm{H}_{\mathrm{t}}\right)$, au milieu du tube $\left(z=\mathrm{H}_{\mathrm{t}} / 2\right)$, et à la base du tube $(z=2 \mathrm{D})$.

L'amplitude maximale du signal filtré en pointe du pieu (Q2D) atteint environ 400 à $450 \mu$ str, ce qui correspond à une contrainte supposée élastique au contact acier-roche d'environ 80 à $90 \mathrm{MPa}$, soit 7,3 à $8,2 \sigma_{c}$ pour une vitesse estimée en pointe de pieu de l'ordre de $2,5 \mathrm{~m} / \mathrm{s}$.

Les essais de battage du tube mince dans le mortier K8 à 4 jours de cure indiquent qu'il devient difficile de battre un tube en acier dans un matériau peu poreux dont la résistance à la compression est de $11 \mathrm{MPa}$. Pour une hauteur de chute de $40 \mathrm{~cm}$, la pénétration semble alterner entre les coups entre une valeur nulle reflétant une fracturation partielle et $1 \mathrm{~mm}$ capitalisant sur la fracturation précédente. Dans cette catégorie de matériau induré, une hauteur de chute modérée $(80 \mathrm{~cm})$ génère un enfoncement du tube de l'ordre de $2 \mathrm{~mm}$ sans que la contrainte en pointe n'excède 80 à $90 \mathrm{MPa}$, soit 7 à $8 \sigma_{c}$ pour une vitesse incidente en tête de pieu de l'ordre de $2,5 \mathrm{~m} / \mathrm{s}$.

Cette conclusion débouche sur l'intérêt qu'il y aurait à réaliser des essais complémentaires de battage dans un matériau dont la résistance serait intermédiaire (entre 11 et $27 \mathrm{MPa})$.

\section{Conclusion générale}

Le problème du battage d'un pieu tubulaire en acier dans un massif induré est complexe et encore faiblement exploré. Les difficultés liées à son caractère dynamique et aux grandes déformations se combinent aux difficultés de modélisation du comportement des massifs indurés. Une approche numérique par éléments finis couplant une modélisation lagrangienne du pieu en mouvement à une modélisation eulérienne du massif en place (approche CEL) permet de caractériser certains aspects de cette complexité. Les résultats de cette approche appliquée à un cas axisymétrique d'étude de pieu de $1,8 \mathrm{~m}$ de diamètre battu dans une roche de $28 \mathrm{MPa}$ de résistance à la compression simple permettent de suivre l'émergence de la plastification du tube en début de pénétration. La plastification naît en pointe, pour se propager vers la tête du pieu. Une qualité supérieure d'acier (S355JR) permet de réduire significativement cette plastification au point qu'une pénétration de 3 fois l'épaisseur 


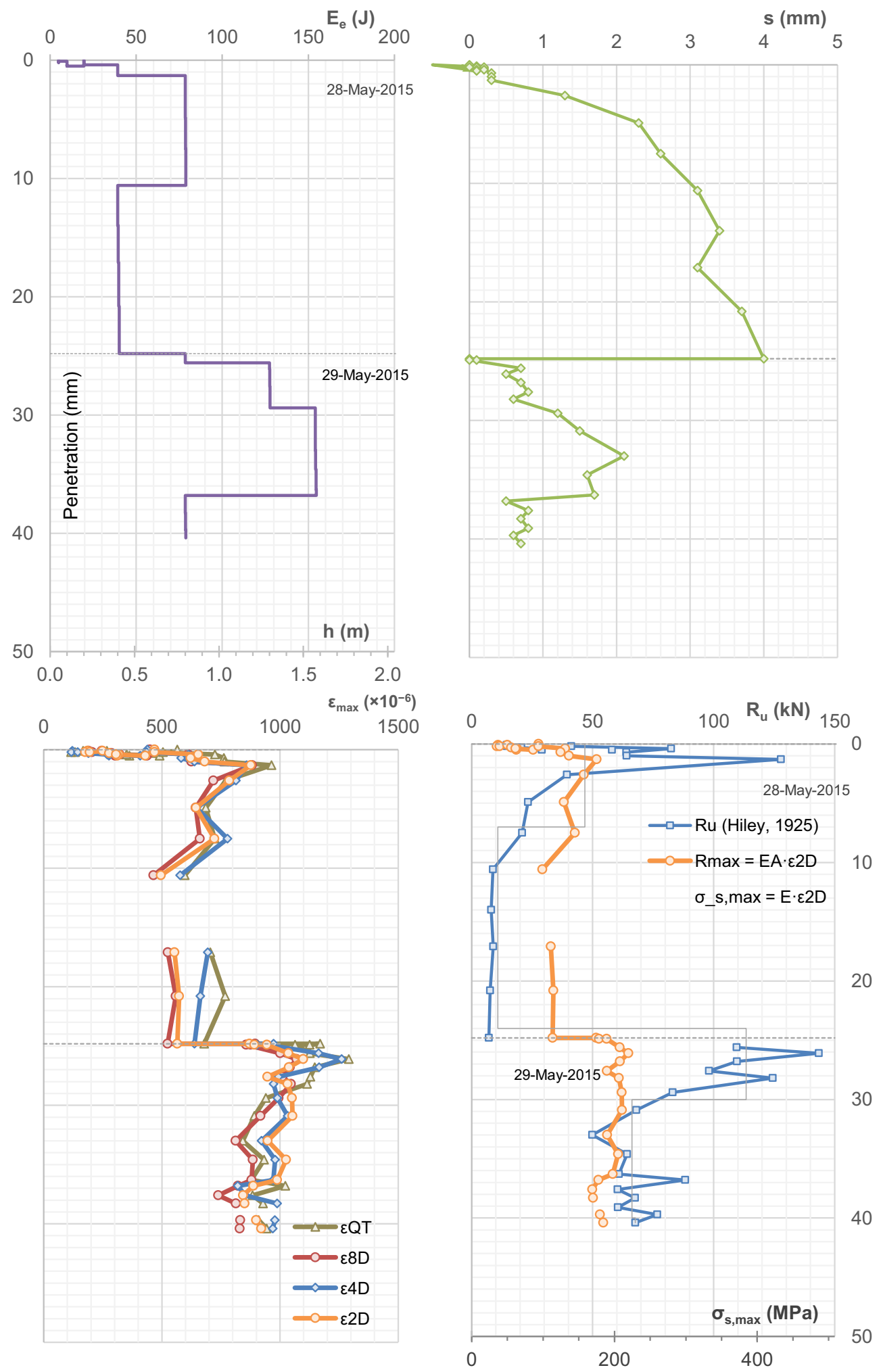

Fig. 21. Battage du pieu court dans le mortier K5 à 26,8 MPa de résistance (a) hauteur de chute/énergie ; (b) refus ; (c) déformation à différents niveaux; (d) résistance du tube à la pénétration et contrainte maximale en pointe (Victor, 2017).

Fig. 21. Short pile driving into 26.8 MPa strength K5 mortar (a) drop height/energy; (b) set; (c) peak strain at different levels; (d) tube penetration resistance and toe peak stress (Victor, 2017). 


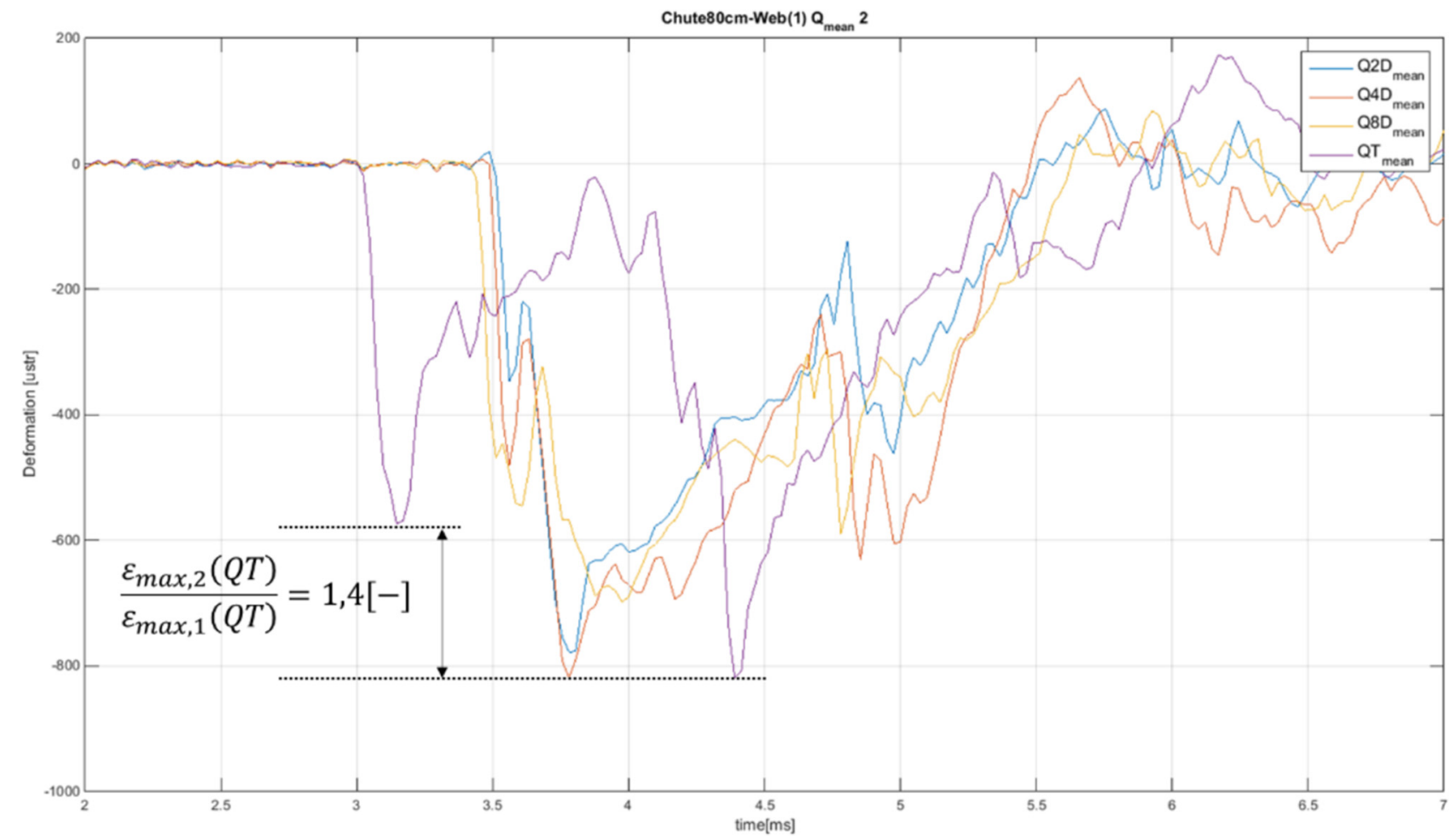

Fig. 22. Déformations mesurées sur le pieu court lors du $2^{\mathrm{e}}$ coup de $80 \mathrm{~cm}$ (UCL, 2016).

Fig. 22. Strains measured in the short pile under the second $80 \mathrm{~cm}$ blow (UCL, 2016).

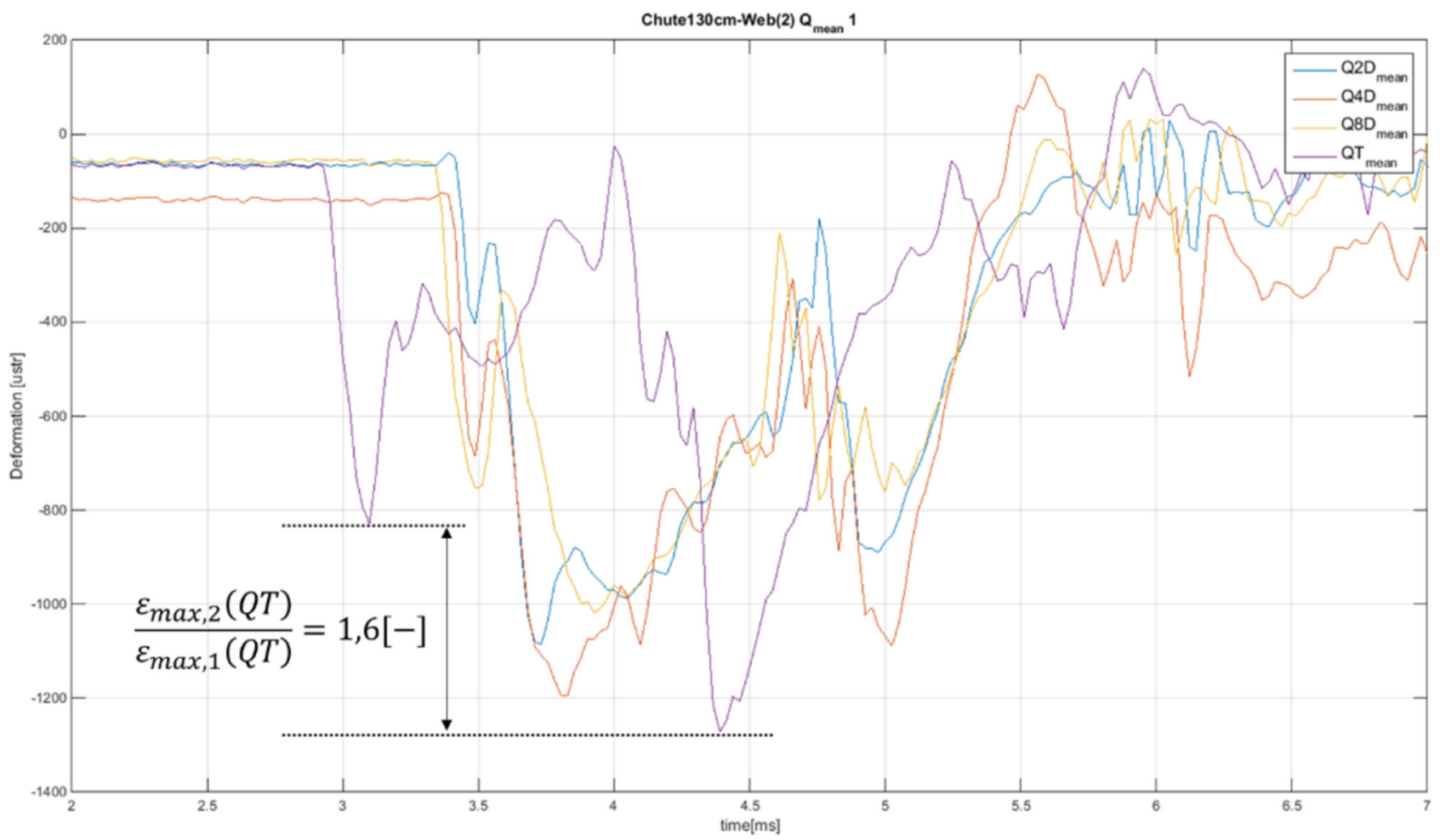

Fig. 23. Déformations mesurées sur le pieu court lors du $1^{\text {er }}$ coup de $130 \mathrm{~cm}$ (UCL, 2016).

Fig. 23. Strains measured in the short pile under the first $130 \mathrm{~cm}$ blow (UCL, 2016). 

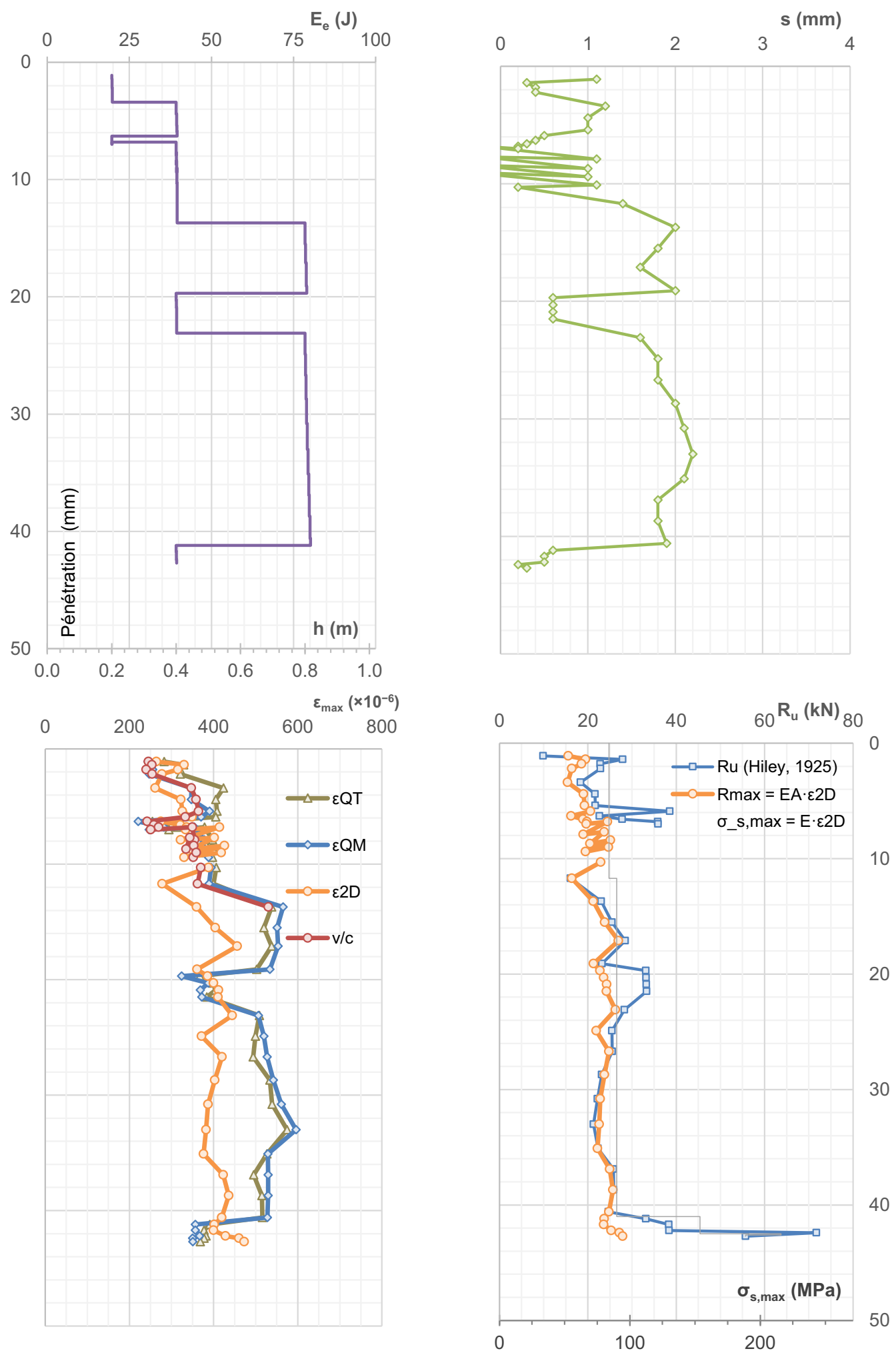

Fig. 24. Battage du pieu mince dans le monolithe K8 de $11 \mathrm{MPa}$ de résistance (a) hauteur de chute/énergie ; (b) refus ; (c) déformation à différents niveaux; (d) résistance du tube à la pénétration/contrainte maximale en pointe (Victor, 2017).

Fig. 24. Driving logs of thin pile into $11 \mathrm{MPa}$ strength 8 monolith (a) drop height/energy; (b) set; (c) peak strain at different levels; (d) tube penetration resistance and toe peak stress (Victor, 2017). 
$\mathrm{du}$ tube pourrait être envisagée dans une roche de cette résistance. Cette conclusion reste préliminaire dans la mesure où elle découle de conditions parfaitement axisymétriques qui excluent tout voilement asymétrique $\mathrm{du}$ tube et toute imperfection géométrique du tube. Elle constitue une borne supérieure à ce qui pourrait être toléré par un pieu réel imparfait battu dans un matériau hétérogène.

Compte tenu des nombreuses incertitudes liées à toute approche numérique complexe, une approche expérimentale a été poursuivie en laboratoire. Trois matériaux synthétisés ont été mis en œuvre sous la forme de monolithes dans lesquels le battage d'un tube en acier inoxydable a été entrepris. Les résultats de ces essais de battage largement instrumentés indiquent que le battage est aisé dans un mortier cellulaire dont la résistance à la compression simple $\sigma_{c}$ n'excède pas $6 \mathrm{MPa}$ mais pratiquement impossible dans un mortier dont la résistance à la compression simple s'approche de $28 \mathrm{MPa}$. Cette dernière observation semble corroborer les résultats de l'approche numérique CEL. Le battage dans un mortier dont la résistance à la compression simple vaut $11 \mathrm{MPa}$ s'est révélée encore faisable sous une hauteur de chute raisonnable.

Les mesures des déformations dans l'acier à différents niveaux dans le tube d'essai ont permis d'identifier un danger d'amplification des contraintes en tête de pieu par interaction de l'onde montante provoquée par la résistance à la pénétration $\mathrm{du}$ massif avec le système de battage. Ce danger augmente avec la résistance du massif et diminue avec la longueur du pieu battu. Les mesures de déformation en pointe permettent de caractériser l'accroissement de la résistance au battage avec la pénétration du tube dans le massif. Les résultats obtenus avec les 3 matériaux mis en œuvre à ce jour indiquent que la résistance unitaire croît d'environ $2 \sigma_{c}$ en surface pour atteindre 6 à $8 \sigma_{c}$ pour une pénétration équivalente à $15-20$ fois l'épaisseur du tube.

Remerciements. Le support de la région Wallone représentée par Monsieur Pascal Lehance de la DG 04 du SPW est vivement apprécié dans la mesure où il a permis de développer un dispositif original de laboratoire et de réaliser les essais dans le cadre du projet de recherche FONDEOLE (Convention $n^{\circ} 1250394$ de subvention pour une recherche industrielle d'intérêt général).

Qu'il me soit permis de remercier le Docteur Sami Hamdi dont les simulations numériques sont mises en valeur ici ainsi que les chercheurs et techniciens Antoine Watterman, KarlHenry Victor, Alex Bertholet, Haythem Gharsallaoui et Mustafa Jafari dont les efforts ont contribué aux résultats de recherche illustrés ici. L'assistance graphique de Maud Archambeau est enfin appréciée.

\section{Références}

Abaqus/Standard Users' Manual, Version 6.14. 2014.

Alves AMM, Lopes FR, Danzinger BR. 2008. Dimensional analysis of the wave equation applied to pile driving. In: Santos JA, ed. Proceedings of the 8 th Stress Wave Conference. Porto: IOS Press, pp. 115-212.
American Petroleum Institute, API. 2011. Geotechnical and foundation design considerations, ANSI/API Recommended Practice 2GEO, ISO 1991-4:2003 (Modified) - Specific requirements for offshore structures, Part 4, 1st edition, April 2001, 103 p.

D'alembert J. Le Rond 1747. Articles extraits de l'Histoire de l'Académie des Sciences et Belles-Lettres de Berlin - Année 1747, Berlin, 1749.

De Saint Venant B. 1867. Mémoire sur le choc de deux barres élastiques. J Math (Liouville): 237-375.

Hamdi S. 2016. Pipe pile driving into rock, Ph.D. Thesis. Louvainla-Neuve, Belgique: Université catholique de Louvain (UCL), $159 \mathrm{p}$.

Hamdi S, Holeyman A. 2015. Numerical modeling of cylindrical cavity expansion in rock mass based on the Hoek-Brown yield criterion, in "International Symposium 60 years of pressuremeters"- Hammamet. Tunisia (ISBN : 978-993812-937-3): 189-196.

Henke S, Grabe J. 2008. Numerical investigation of soil plugging inside open-ended piles with respect to the installation method. Acta Geotechnica 3: 215-223. DOI: 10.1007/s11440-008-0079-7.

Hoek E, Brown ET. 1980. Empirical strength criterion for rock masses. J Geotech Eng Div ASCE 106: 1013-1035.

Hoek E, Brown ET. 1988. The Hoek-Brown failure criterion - a 1988 update. In : Curran JC, ed. Proc. 15th Canadian Rock Mech. Symp, Toronto: Dept. Civil Engineering, University of Toronto, pp. 31-38.

Hoek E, Carranza-Torres C, Corkum B. 2002. Hoek-Brown Failure Criterion -2002 Édition, Proc. NARMS Conference, Toronto, pp. 267-273.

Holeyman A. 1984. Contribution à l'étude du comportement transitoire non-linéaire des pieux pendant leur battage. Thèse de doctorat, Université Libre de Bruxelles, Avril, 584 p.

Holeyman A. 1992. Technology of pile dynamic testing. In : Barends F, ed. Application of Stress-Wave Theory to Piles. Rotterdam: Balkema, pp. 195-215.

Holeyman A, Whenham V. 2015. Axial non-linear dynamic soil-pile interaction. In : Belhaq M, ed. Structural nonlinear dynamics and diagnosis, pp. 305-334 (ISBN : ISBN 978-3-319-19851-4). Available from: https://doi.org/10.1007/978-3-319-19851-4.

Holeyman A, Whenham V. 2017. Critical review of the hypervib1 model to assess pile vibro-drivability. Geotech Geol Eng: an international journal 2017(2): 1-19. Available from: https://doi. org/10.1007/s10706-017-0218-8.

Holeyman A, Bertin R, Whenham V. 2013. Impedance of pile shafts under axial vibratory loads. Soil Dyn Earthq Eng 44: 115-126. Available from: https://doi.org/10.1016/j.soildyn.2012.09.006.

Holeyman AE. 1988. Modelling of dynamic behaviour at the pile base. Proceedings of the 3rd International Conference on the Application of Stress-Wave Theory to Piles. Ottawa, Canada, pp. $174-185$.

Isaacs DV. 1931. Reinforced concrete pile formulae, Paper No. 370, Transactions of the Institution of Engineers, Australia, Vol. XII, pp. 371-399.

Jablonski J, Carlucci P, Thyagarajan R, Nandi B, Arata J. 2012. Simulating underbelly blast events using Abaqus/Explicit - CEL, SIMULIA Customer Conference.

Marinos V, Marinos P, Hoek E. 2005. The geological strength index, applications and limitations. Bull Eng Geol Environ 64: 55-65.

Norme Française NF EN 1997-1, Eurocode 7. 2005. Calcul géotechnique, Partie 1 : Règles générales, $146 \mathrm{p}$.

Norme Française NF EN 1997-1/NA. 2006. Annexe nationale à la NF EN 1997-1, Eurocode 7, 11 p. 
Norme Française NF P 94-262. 2012. Justification des ouvrages géotechniques, Normes d'application nationale de l'Eurocode 7, Fondations Profondes, $205 \mathrm{p}$.

Priest S. 2005. Determination of shear strength and three-dimensional yield strength for the Hoek-Brown criterion. Rock Mech Rock Eng 38: 299-327.

Puech A, Poulet D, Boisard P. 1990. A procedure to evaluate pile drivability in the difficult soil conditions of the southern part of the Gulf of Guinea, Offshore Technology Conference Paper OTC 6237, pp. 327-334.

Qiu G, Henke S, Grabe J. 2011. Application of a coupled EulerianLagrangian approach on geomechanical problems involving large deformations. Comput Geotech 38: 30-39.
Ribacchi R. 2000. Mechanical tests on pervasively jointed rock material: insight into rock mass behaviour. Rock Mech Rock Eng 33 (4): 243-266.

Smith EAL. 1960. Pile driving analysis by the wave equation. $J$ Soil Mech Found Div (ASCE) 6: 35-61.

UCL. 2016. Rapport interne d'avancement du projet de recherche FONDEOLE, Convention $n^{\circ} 1250394$ de subvention par la Région Wallonne pour une recherche industrielle d'intérêt général, janvier 2016, 235 p.

Upwind - Integrated Wind Turbine Design (2010), Project funded by the European Commission under the 6th (EC) RTD Project No. 019945 (SE6)

Victor KH. 2017. Communication personnelle.

Citation de l'article : Alain Holeyman. Battage de pieux métalliques dans la roche. Rev. Fr. Geotech. 2017, $153,1$. 